\title{
Baseline results of a living systematic review for COVID-19
}

\section{funded research projects [version 1; peer review: 2 approved]}

\author{
Alice Norton (D1,2, Adrian Bucher1, Emilia Antonio (D1)1,3, Nicole Advani1,3, \\ Henrike Grund1, Sheila Mburu1, Emma Clegg1', Genevieve Boily-Larouche4, \\ Morgan Lay5, Gail Carson², Marta Tufet Bayona1
}

\author{
1 UK Collaborative on Development Research, London, UK \\ ${ }^{2}$ GloPID-R Secretariat, Centre for Tropical Medicine and Global Health, University of Oxford, Oxford, UK \\ ${ }^{3}$ Centre for Tropical Medicine and Global Health, University of Oxford, Oxford, UK \\ ${ }^{4}$ Institute of Infection and Immunity, Canadian Institutes of Health Research, Hamilton, Canada \\ ${ }^{5}$ Institute of Population and Public Health, Canadian Institutes of Health Research, Toronto, Canada
}

V1 First published: 08 Sep 2020, 5:209

https://doi.org/10.12688/wellcomeopenres.16259.1

Second version: 18 Dec 2020, 5:209

https://doi.org/10.12688/wellcomeopenres.16259.2

Third version: $01 \mathrm{Apr}$ 2021, 5:209

https://doi.org/10.12688/wellcomeopenres.16259.3

Fourth version: $01 \mathrm{Jul}$ 2021, 5:209

https://doi.org/10.12688/wellcomeopenres.16259.4

Fifth version: 22 Mar 2022, 5:209

https://doi.org/10.12688/wellcomeopenres.16259.5

Sixth version: 28 Jul 2022, 5:209

https://doi.org/10.12688/wellcomeopenres.16259.6

Seventh version: 06 Sep 2022, 5:209

https://doi.org/10.12688/wellcomeopenres.16259.7

Eighth version: 16 Nov 2022, 5:209

https://doi.org/10.12688/wellcomeopenres.16259.8

Latest published: 01 Feb 2023, 5:209

https://doi.org/10.12688/wellcomeopenres.16259.9

\section{Abstract}

Background: The coronavirus disease 2019 (COVID-19) has resulted in an unprecedented research response, demonstrating exceptional examples of rapid research and collaboration. There is however a need for greater coordination, with limited resources and the shifting global nature of the pandemic resulting in a proliferation of research projects underpowered and unable to achieve their aims.

Methods: The UK Collaborative on Development Research (UKCDR) and Global Research Collaboration for Infectious Disease Preparedness (GloPID-R), two funder coordination groups have collaborated to develop a live database of funded research projects across the world relating to COVID-19. Drawing data continually from their members and further global funding bodies, as of $15^{\text {th }}$ July 2020 the database contains 1,858 projects, funded by 25 funders, taking place across 102 countries. To our knowledge it is one of the most

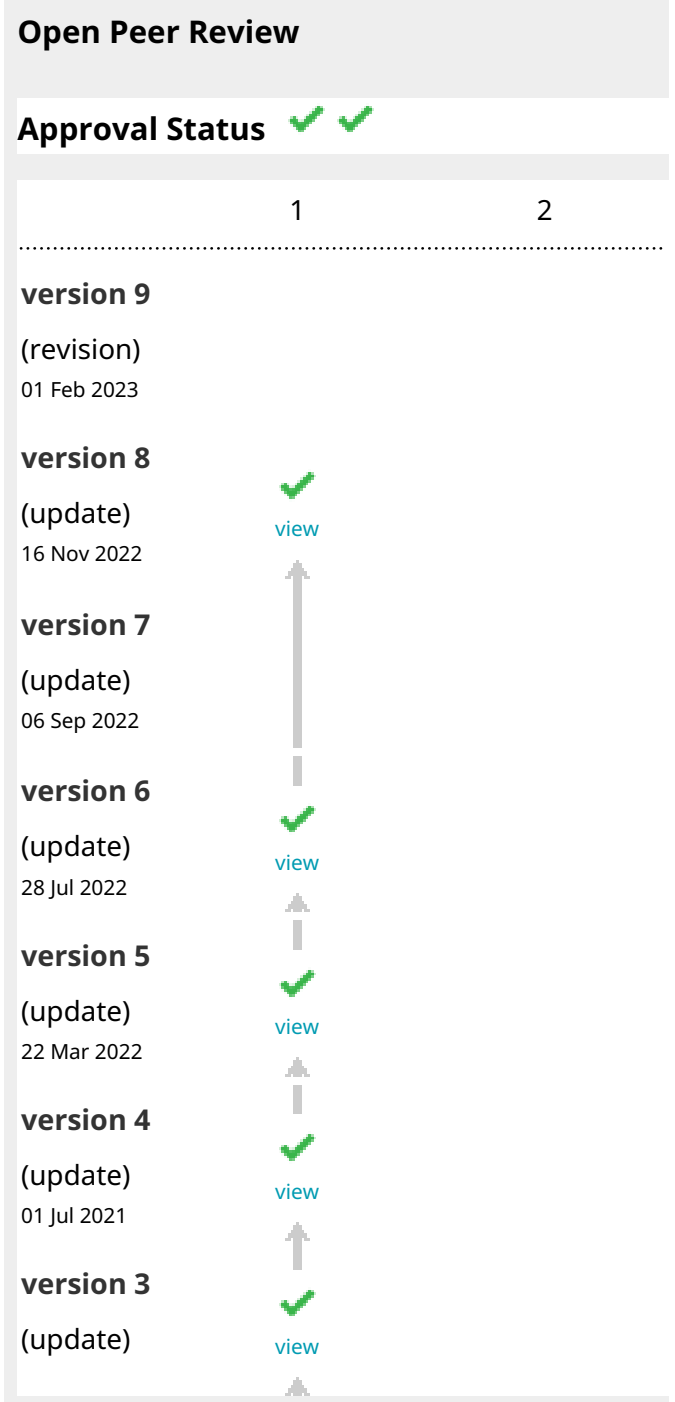


comprehensive databases, covering a wide breadth of research disciplines. The database is aligned to the World Health Organisation (WHO) Global Research Roadmap: 2019 Novel Coronavirus. It is being used by the WHO, governments and multi-lateral policy makers, research funders and researchers.

This living systematic review aims to supplement the database by providing an open accessible and frequently updated resource summarising the characteristics of the COVID-19 funded research portfolio. Both descriptive and thematic analysis will be presented and updated frequently to aid interpretation of the global COVID-19 funded research portfolio.

Results: In this baseline analysis we provide the first detailed descriptive analysis of the database and focus our thematic analysis on research gaps, study populations and research locations (with a focus on resource-limited countries).

Conclusions: This living systematic review will help both funders and researchers to prioritise resources to underfunded areas where there is greatest research need and facilitate further strategic collaboration.

\section{Keywords}

Living systematic review, COVID-19, Coronavirus, research funding, coordination, global health policy

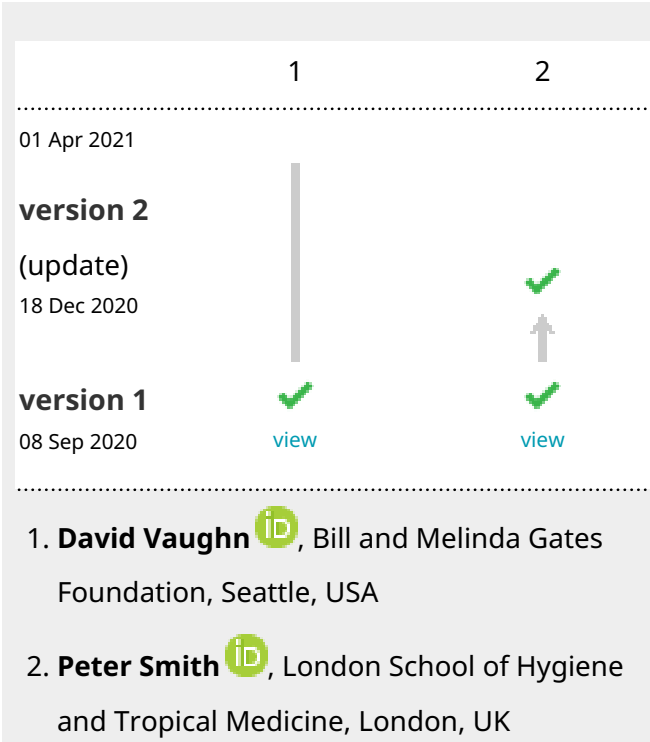

Any reports and responses or comments on the article can be found at the end of the article.

\section{This article is included in the Coronavirus} (COVID-19) collection.

\section{Corresponding author: Alice Norton (alice.norton@ndm.ox.ac.uk)}

Author roles: Norton A: Conceptualization, Data Curation, Funding Acquisition, Investigation, Methodology, Project Administration, Supervision, Validation, Writing - Original Draft Preparation; Bucher A: Data Curation, Formal Analysis, Investigation, Methodology, Visualization, Writing - Original Draft Preparation; Antonio E: Data Curation, Formal Analysis, Investigation, Methodology, Visualization, Writing - Original Draft Preparation; Advani N: Data Curation, Formal Analysis, Investigation, Methodology, Visualization, Writing Original Draft Preparation; Grund H: Data Curation, Project Administration, Resources, Software, Writing - Review \& Editing; Mburu S: Data Curation, Writing - Review \& Editing; Clegg E: Data Curation, Software, Writing - Review \& Editing; Boily-Larouche G: Methodology, Validation, Writing - Review \& Editing; Lay M: Methodology, Validation, Writing - Review \& Editing; Carson G: Methodology, Resources, Validation, Writing - Review \& Editing; Tufet Bayona M: Conceptualization, Funding Acquisition, Resources, Validation, Writing - Review \& Editing

Competing interests: GB-L and AML are both employed by the Canadian Institutes of Health Research funder and undertook this work as a contribution to GloPID-R's work. The other authors declare no competing interests.

Grant information: The COVID CIRCLE initiative is funded by Wellcome, UK DHSC and UKRI. COVID CIRCLE is also supported by core funding to UKCDR from DFID, UK BEIS, UKRI, UK DHSC, Wellcome and DEFRA and by in-kind contributions from the GloPID-R Secretariat funded by the European Union's Horizon 2020 Research and Innovation Programme under grant agreement 643434.

Copyright: $\odot 2020$ Norton A et al. This is an open access article distributed under the terms of the Creative Commons Attribution License , which permits unrestricted use, distribution, and reproduction in any medium, provided the original work is properly cited.

How to cite this article: Norton A, Bucher A, Antonio $E$ et al. Baseline results of a living systematic review for COVID-19 funded research projects [version 1; peer review: 2 approved] Wellcome Open Research 2020, 5:209

https://doi.org/10.12688/wellcomeopenres.16259.1

First published: 08 Sep 2020, 5:209 https://doi.org/10.12688/wellcomeopenres.16259.1 


\section{Introduction}

Researchers and research funders in global health have been preparing for a pandemic such as that caused by severe acute respiratory syndrome coronavirus 2 (SARS-CoV-2) for decades; however, the urgency and global scale of the research needs and response has been difficult to respond to and coordinate. Research funders have rapidly supported repurposing of existing studies and launched rapid funding calls to support research into the most pressing needs. Lessons in expediting research have been learnt from undertaking research in the recent North Kivu Ebola outbreak and West Africa Ebola, Zika and SARS epidemics, however the truly global nature of the coronavirus disease 2019 (COVID-19) pandemic has led to unprecedented needs and challenges for coordination.

The World Health Organisation (WHO) triggered a rapid response, building on the $\mathrm{R} \& \mathrm{D}$ Blueprint ${ }^{1}$, and co-organised the Global Research and Innovation Forum: Towards a Research Roadmap for the 2019 Novel Coronavirus meeting with the Global Research Collaboration for Infectious Disease Preparedness (GloPID-R) on February 11-12, 2020 at which over 400 global experts identified research priorities for COVID-19. In March 2020, the WHO released the WHO Coordinated Global Research Roadmap: 2019 Novel Coronavirus (WHO Roadmap) ${ }^{2}$ to coordinate and accelerate the global research response against the identified priorities. The WHO Roadmap is an unprecedented galvanizing document for global research collaboration. This project builds on this to help shepherd the global response.

In a joint effort to further coordinate and synergise the funding of research to address the WHO Roadmap identified priority areas, the UK Collaborative on Development Research (UKCDR) partnered with GloPID-R to launch the COVID-19 Research Project Tracker $^{3}$ (the tracker) on April 3, 2020. The tracker is a live database of funded research projects across the world related to the current COVID-19 pandemic. It includes both newly funded research projects and repurposed research projects across all disciplines and categorises them against the mid- to longterm research and development priorities and sub-priorities identified in the WHO Roadmap. Mapping of projects as soon as funding is announced allows visibility of the funded research portfolio well in advance of any outputs such as publications.

The UKCDR Epidemics Preparedness and Response Funders Group $^{4}$ and the GloPID-R Key Funders group have each been meeting at least fortnightly during the pandemic to strengthen UK and global COVID-19 research funding coordination activities respectively, informed by the data and analysis from the tracker. Several members of both organisations have recently launched calls for research on COVID-19 in low and middle-income country (LMIC) settings. There is a particular concern that due to the resource limitations in LMICs an uncoordinated approach could potentially lead to unaddressed local research needs, failure of research to inform policy or unsustainable research capacity to respond to future outbreaks. The UKCDR and GloPID-R funders groups have agreed to align to further strengthen their response by agreeing to a set of Funder Principles for supporting high-quality research for the most pressing global needs in epidemics and pandemics ${ }^{5}$ and with the formation of a new jointly hosted initiative for COVID-19 Research Coordination and Learning (COVID CIRCLE), encompassing the tracker and with a particular focus on resource-limited settings ${ }^{5}$.

As part of the COVID CIRCLE initiative, this living systematic review has been established to regularly update and incorporate newly funded research projects as they become available and review their alignment to the WHO Roadmap priorities. A living systematic review (LSR) is needed due to the rapidly expanding number of funded research projects and the importance of the review to inform funding decision making. Here we present the results of the baseline review of all research projects within the tracker as of $15^{\text {th }}$ July 2020 and a descriptive and thematic analysis to aid interpretation of the global COVID-19 funded research portfolio.

\section{Methods}

\section{Protocol for LSR}

The LSR protocol outlined herein was prospectively designed. Due to the rapid need for this project to be conducted to inform research responses during the pandemic, data extraction commenced before the protocol could be formally registered with PROSPERO. The protocol is outlined in this paper.

\section{Rationale for use of living method}

Funding bodies have responded rapidly to the COVID-19 pandemic through repurposing existing grants and rapidly funding projects with both rolling and one-off funding calls. This has resulted in new research projects being funded at short intervals necessitating a living systematic review for this work. The regular update of this review will help coordinate ongoing researcher and funder responses.

\section{Eligibility criteria}

All research projects funded by any research funder around the world (including regional funding organisations, national research funders and non-profit/ philanthropic organisations), with a focus on COVID-19 were eligible for inclusion in this analysis. This includes data from all types of research activities and was not limited to just biomedical and health research. Furthermore, this analysis includes grants identified by funders as having been repurposed to address COVID-19 research priorities.

\section{Information sources and search strategy}

The database and subsequent analysis make use of data from publicly announced COVID-19 research grants and were obtained using one of two methods. The more common of the two methods to obtain data was through direct communication with research funders by requesting the completion of a template spreadsheet (Extended data $1^{19}$ ). These requests were made to UKCDR and GloPID-R funder groups members ${ }^{6,7}$ on a fortnightly basis (as part of funder coordination meetings) and to wider funder contacts beyond these groups. Alternatively, data were also obtained from online databases belonging to research funders using "COVID" and/or "coronavirus" as search terms (see Extended Data $2^{19}$ ). The tracker remains open to the 
submission of new funding data relating to COVID-19 from any global funder at any time. Screening of submitted data occurs on a weekly basis.

Though the set of data fields varied between funders, the data fields presented in Table 1 were considered a priority for the purposes of the tracker and subsequent analyses:

\section{Update schedule}

All figures will be updated on a three-monthly basis, when the discussion will also be revised to reflect any changes and trends over time. This living systematic review will continue to be updated for the duration of the COVID CIRCLE initiative funding. The frequency of screening will not be reduced for the duration of COVID CIRCLE, although updates will only continue where new grants are included.

\section{Manually coded data fields}

Data entry of additional manually classified variables was completed by one reviewer with each variable cross-checked by a second reviewer. Abstracts not in English (French and German) were coded by project team members fluent in those languages. Projects were coded to the following classifications:

\section{WHO medium-long term research priorities and sub-priorities} Projects were assigned to one or more WHO priority areas of primary focus (Extended data $3^{19}$ ). An assignment of 'N/A' was made where: information provided was insufficient for classification; funds were allocated for research administration; or where projects clearly fell outside the WHO broad priority areas. Subsequently, projects were assigned to appropriate WHO sub-priority area(s). The assignment of 'N/A' was made if insufficient information limited further sub-categorisation or the projects fell outside the WHO sub-priority areas. In addition, suitable secondary priority area(s) with corresponding subpriority(ies) were determined for those projects that significantly addressed other priority areas. Hence, projects were assigned with multiple primary and/or secondary WHO priority ad subpriority areas of research focus. The priority list will be updated if future iterations of the WHO Roadmap are released.

\section{Emergent categories for research falling outside the WHO priority classification}

For those projects that were not considered as addressing any of the WHO Research Priorities, they were assigned 'N/A' and new sub-priorities were developed and assigned on an initial data set of 400 projects. An inductive approach was used to develop new codes that emerged from the funded research and themes were confirmed through an iterative process through the projects in this baseline assessment. Six new sub-priority codes were defined under the social science priority (mental health; digital health; policy and economics; education; logistics and food security). A new priority focusing on the environmental impacts of COVID-19, was developed as well. All newly identified categories were validated using the full baseline dataset.

\section{COVID-19 Research Priorities for LMICs}

Research projects involving LMICs were additionally assessed for their alignment with their research priorities identified in

Table 1. Priority data fields for the UK Collaborative on Development Research (UKCDR) and Global Research Collaboration for Infectious Disease Preparedness (GloPID-R) tracker and analysis. The latest and previous versions of this table are available as extended data ${ }^{19}$.

\begin{tabular}{|l|l|}
\hline Data Field & Definition \\
\hline Abstract & $\begin{array}{l}\text { Scientific summary of the project } \\
\text { Amount awarded } \\
\text { Total amount awarded by the responsible funder for the duration of } \\
\text { the project (with currency stated) }\end{array}$ \\
\hline $\begin{array}{l}\text { Country(-ies) where studies are } \\
\text { being conducted }\end{array}$ & All countries where research is being conducted \\
\hline Funder(s) & The names of all funding organisations (including co-funding) \\
\hline Lead institution & $\begin{array}{l}\text { The name of the organisation that holds the grant and is leading the } \\
\text { research }\end{array}$ \\
\hline Local implementing partner(s) & $\begin{array}{l}\text { The name of any partner institutions located in the country(-ies) } \\
\text { where the study is being conducted }\end{array}$ \\
\hline Principal investigator & $\begin{array}{l}\text { Name of the awarded project's lead investigator based at the lead } \\
\text { institution (primarily used for project de-duplication) }\end{array}$ \\
\hline Project ID/reference number & $\begin{array}{l}\text { Any unique reference number / project ID assigned by the funder } \\
\text { organisation to this project (primarily used for project de-duplication) }\end{array}$ \\
\hline Project title & \begin{tabular}{l} 
Title of the research project \\
\hline Start/end date
\end{tabular} \\
\hline
\end{tabular}


a collaborative study conducted by the UKCDR, African Academy of Sciences (AAS) and the Global Health Network (TGHN) in May, 20208,9. This study, which determined globally relevant COVID-19 research priorities with a specific focus on less-resourced countries, was based on earlier work by the AAS to determine the COVID-19 research priorities for Africa ${ }^{8}$ and the mid- to long-term research priorities summarized in the WHO Research Roadmap ${ }^{2}$. The study findings, published in August 2020, outline existing WHO research priorities which require greater research emphasis and new research priority areas not captured in the WHO Roadmap or identified in the AAS survey (Extended data $4^{19}$ ). Each funded research project involving LMICs was assigned to one of the new categories outlined in Supplementary material 2 or noted if it fell outside both the new AAS priorities and the new priorities identified by the UKCDR/AAS/TGHN study.

\section{Cross-cutting themes}

During the data coding process, a number of cross-cutting themes identified by the project team for analysis. For this baseline assessment, the following additional variables were identified: repurposed projects; capacity strengthening and innovation.

\section{Health Research Classification System}

To capture information on the type of research taking place in relation to COVID-19 with a stable classification system that is comparable to research on other health topics, the projects included in the tracker were also assessed against the research activity codes outlined by the Health Research Classification System (HRCS) - a classification system developed by UK Clinical Research Collaboration and used by health research funders around the world to classify "the full spectrum of biomedical and health research - from basic to applied - across all areas of health and disease." ${ }^{\prime \prime}$. While not all of the data in the tracker have been classified against the HRCS research activity codes yet ( 821 of 1,858 coded), it is anticipated that all research projects will be classified appropriately with future updates to this analysis. For those projects that have already been coded against the HRCS, each project was assigned with an activity code or 'N/A' either when the research activity fell outside of those listed under the HRCS codes or where there was not enough project information to make an assessment.

\section{Study population}

A study population categorisation structure was proposed using an inductive approach on an initial data set of 400 projects and validated using the full data set, allowing the categories to be specific to the populations represented in the funded research.

For the purposes of this analysis, a hierarchical categorisation system was produced to examine the study populations of the research projects included in the tracker. At the highest levels, research projects are assessed on whether they involve animal populations, human populations, literature reviews, policy analysis or only focus on the virus itself. Research projects focused on human populations, were classified against three additional sub-categories. Table 2 outlines the categories, sub-categories and levels in full.

\section{Synthesis of results}

In light of the stated aim of the tracker (and subsequent analyses) to provide an overview of trends in funded COVID-19 research, descriptive and comparative analyses are used in this analysis to present the information included in the version of the tracker updated July $15^{\text {th }} 2020$.

The data used for this analysis can be obtained from the COVID-19 Research Project Tracker page on UKCDR's website, as mentioned in the data availability statement. Data on the tracker (and subsequent analyses) will continue to be updated as more data becomes available and are obtained by the project team.

The charts and figures produced in this analysis were produced using Microsoft Office (Office 365 versions of Excel and PowerPoint) and Tableau (version 2020.2)

\section{Limitations of the data}

Among the main challenges of the analysis is the varying degrees of completeness of data across funders which hindered assignment of projects to broad priority and sub-priority areas where the qualitative details of projects provided were insufficient. Therefore, the assigned priority areas may have failed to completely capture all aspects of the projects relevant to the WHO Roadmap. The same can be said for any value that was assigned to a given research project by the project team, including the study population and type of research activity. The data validation process by reviewers with expertise in global health research, policy, and funding outlined in the Project Selection section was used to address this and ensure that any assigned value was as accurate as possible, given the information provided.

As far as funding amounts are concerned, this analysis is limited in providing a holistic picture of trends in COVID-19 research funding data was available from 10 of 25 funders (91.4\% of all projects). However, as the analysis makes use of all possible information that is publicly available, it can still be considered as being as comprehensive as is possible.

At a higher level, the comprehensiveness of the tracker is limited to the funders that have either provided data for the tracker or had their data extracted from online sources (if available). In this respect, there were challenges in engaging with (and obtaining data from) health research funders beyond existing networks either due to a lack of contacts or capacity from funders to contribute to the project (especially for funders whose data is not in English). Few funders have yet identified or made available details on grants repurposed towards COVID-19 to date.

\section{Risk of bias}

This LSR of funded COVID-19 research projects uses descriptive and thematic analysis to summarise the scope of funded COVID-19 research projects. No attempts are made to assess the quality of individual studies or whether the studies meet their objectives. The potential sources of bias with project 
Table 2. Study population categorisation system. The latest and previous versions of this table are available as extended data ${ }^{19}$.

\begin{tabular}{|c|c|c|}
\hline Level & Category & Sub-category \\
\hline \multirow{6}{*}{1} & \multirow{6}{*}{ Population } & Animal population \\
\hline & & Human population \\
\hline & & Literature reviews \\
\hline & & Policy \\
\hline & & Virus \\
\hline & & Other \\
\hline \multirow{4}{*}{2} & \multirow{4}{*}{ Human sub-population } & Adults \\
\hline & & Adults- women \\
\hline & & Adolescents \\
\hline & & Children \\
\hline \multirow{10}{*}{$3 a$} & \multirow{10}{*}{$\begin{array}{l}\text { Population group- vulnerable } \\
\text { populations }\end{array}$} & Care home patients \\
\hline & & Disabled \\
\hline & & Domestic Violence Victims \\
\hline & & Elderly \\
\hline & & High risk individuals (defined as such in the study) \\
\hline & & LGBTQI+ community \\
\hline & & Minority communities (defined as such in the study) \\
\hline & & Neonates \\
\hline & & Pregnant women \\
\hline & & Refugees \\
\hline \multirow{6}{*}{$3 b$} & \multirow{6}{*}{$\begin{array}{l}\text { Population group- Frontline } \\
\text { workers - Healthcare workers }\end{array}$} & Care home staff \\
\hline & & Doctors \\
\hline & & Informal \\
\hline & & Nurses \\
\hline & & Paramedics \\
\hline & & Social care workers \\
\hline \multirow{3}{*}{$3 c$} & \multirow{3}{*}{$\begin{array}{l}\text { Population group- Frontline } \\
\text { workers - Non-healthcare }\end{array}$} & Firefighters \\
\hline & & Sanitation \\
\hline & & Volunteers \\
\hline \multirow{4}{*}{4} & \multirow{4}{*}{ COVID-19 infection status } & Negative \\
\hline & & Negative - Recovered \\
\hline & & Positive \\
\hline & & Positive - Severe \\
\hline
\end{tabular}

LGBTQI+ - Lesbian, gay, bisexual, transgender, queer and intersex, COVID-19 - Coronavirus disease 2019 
selection, quality of data reviewed, and data extraction and classification are addressed by robust fortnightly searches, template completion by funders and independent assessment and review during project classification respectively, as mentioned in the Information Sources and Search Strategy.

While the intention of the tracker and subsequent analyses are to provide as comprehensive a picture as possible of the COVID-19 research landscape, the data obtained for the tracker is more likely to be derived from funders of research that are members of UKCDR (all UK and broad disciplinary focus) and/or GloPID-R (global membership spanning HICs to LICs with a biomedical focus). This would likely skew the results to show that more research being funded from these organisations and reflect trends in their respective portfolios (in terms of location, research focus and research activity type) than may necessarily be the case of the landscape more generally. The funders whose data are included in the tracker currently are based in just 13 (high-income) countries. We are anticipating funding data from several ongoing calls by funders based in LMICs.

\section{Results}

Project selection

In total, 2,007 projects were assessed against the eligibility criteria outlined in the methods and 149 were excluded for being duplicate projects or failing to meet the eligibility criteria as they were not related to COVID-19 (PRISMA Flow Diagram provided in Figure 1). The remaining 1,858 projects were assigned to the manually coded data fields by nine

\section{PRISMA 2009 Flow Diagram}
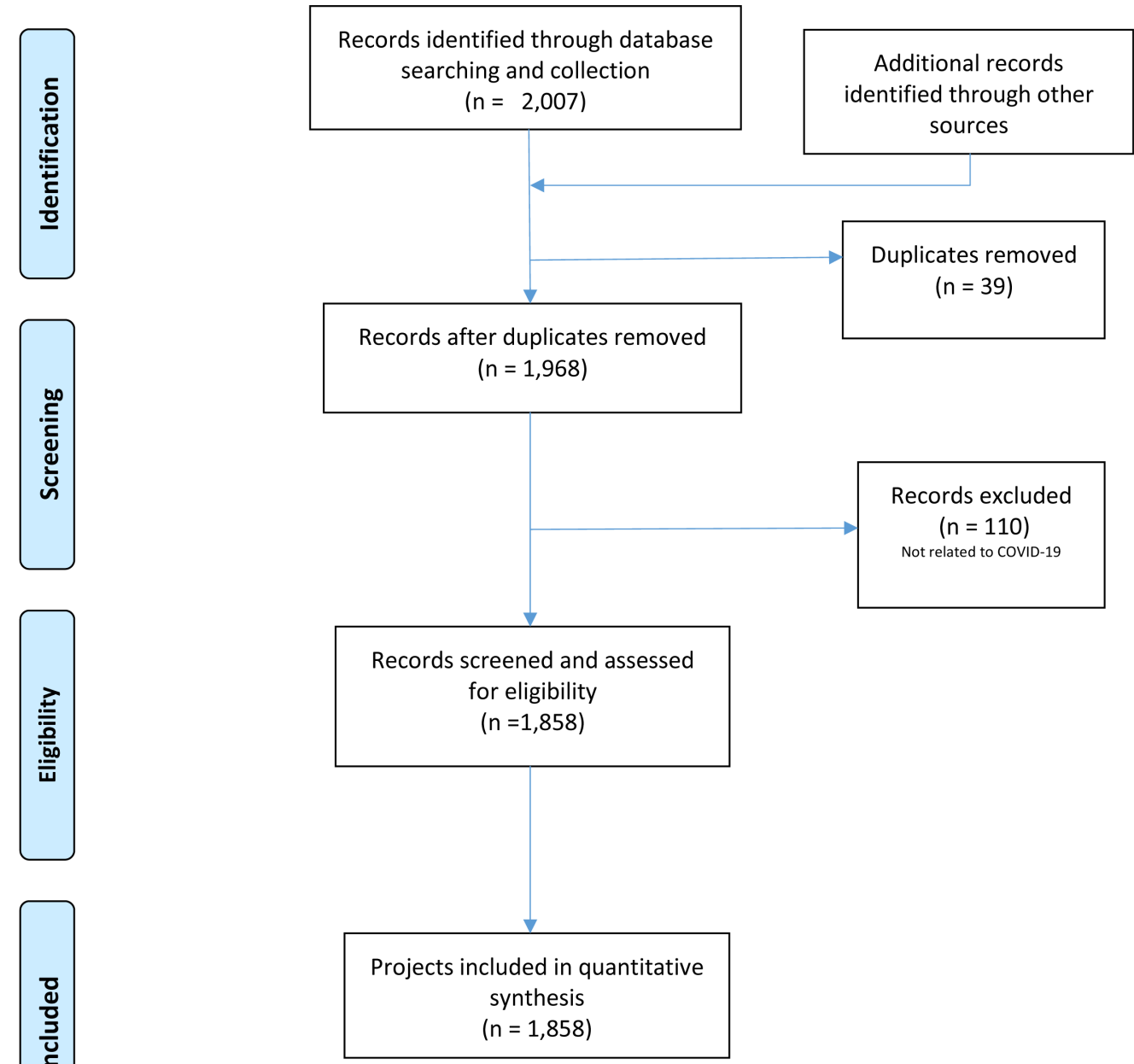

Figure 1. PRISMA flow diagram. The latest and previous versions of this figure are available as extended data ${ }^{19}$. 
project team members before being validated by an independent reviewer not involved with the initial screening and assigning process. All reviewers had broad expertise in global health research, policy, and funding.

\section{Project characteristics}

Summaries of the characteristics of the 1,858 projects included in the latest version of the tracker are provided in the discussion of the results (below) which breaks down the projects by:

- Funder;

- Priority and sub-priority areas;
- Location;

- Activity type;

- Study population.

A full list of the projects is provided as underlying data ${ }^{18}$.

Project funder. The 1,858 projects included in the latest version of the tracker comprises of data compiled from 25 research funders based in 13 different countries (Figure 2). Of these funders, nearly half of the total number of research projects on the tracker $(46.2 \%)$ were awarded by funders based in the UK,

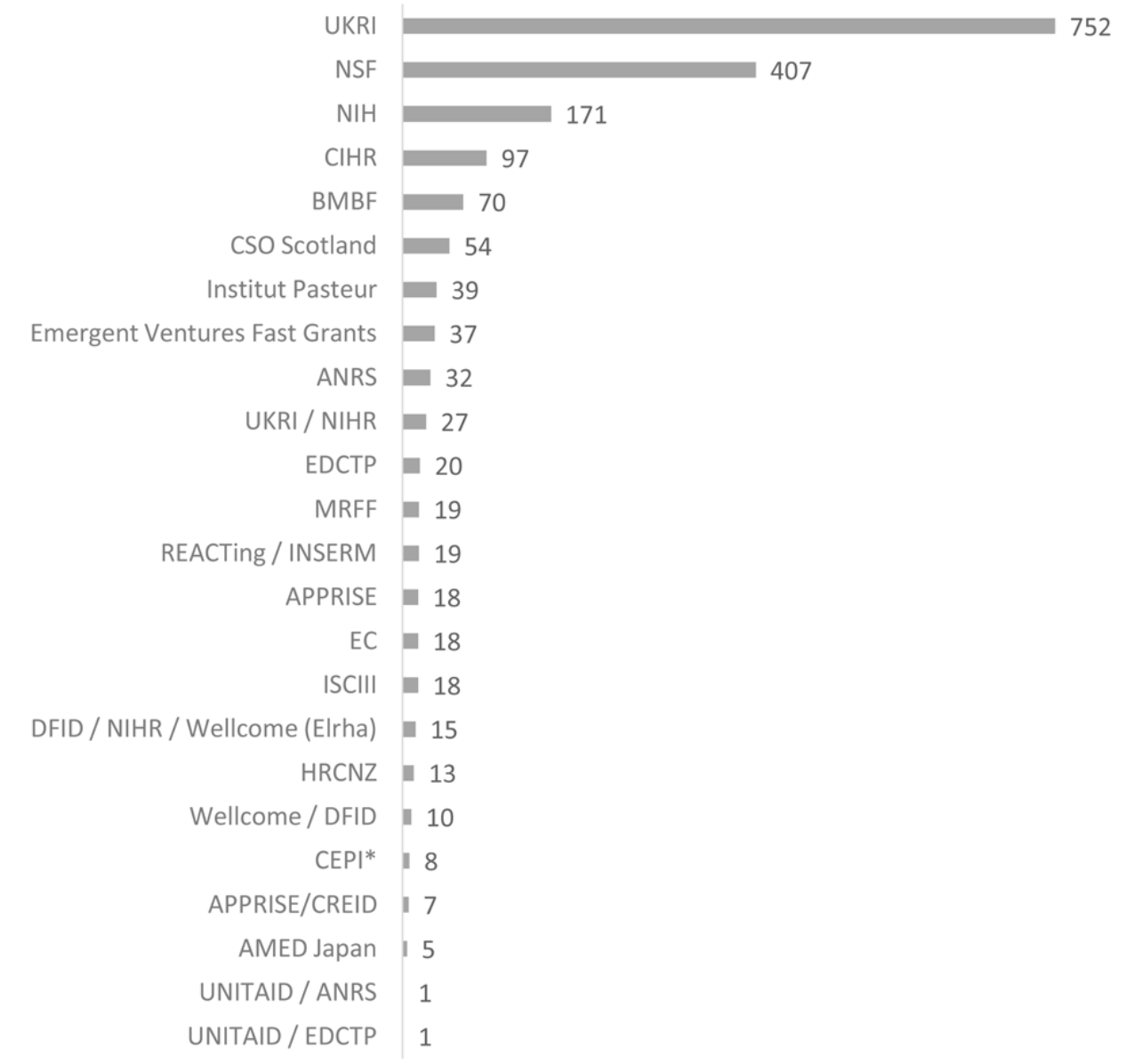

Note: Multiple funders on the same row indicates co-funded projects and are counted separately to instances where one of the funders appear elsewhere.

Figure 2. Number of projects by research funder. The latest and previous versions of this figure are available as extended data ${ }^{19}$. * Projects funded by CEPI are financed by multiple funders in addition to their own funding calls. Abbreviations and acronyms: AMED Japan - Japan Agency for Medical Research and Development; ANRS - French Agency for Research on AIDS and Viral Hepatitis; APPRISE - Australian Partnership for Preparedness Research on Infectious Disease Emergencies; BMBF - Federal Ministry of Education and Research (Germany); CEPI - Coalition for Epidemic Preparedness Innovations; CIHR - Canadian Institutes of Health Research; CREID - Centre of Research Excellence in Emerging Infectious Diseases; CSO Scotland - Chief Scientist Office Scotland; DFID - Department for International Development (UK); EC - European Commission; EDCTP - European \& Developing Countries Clinical Trials Partnership; HRCNZ - Health Research Council of New Zealand; INSERM - National Institute of Health and Medical Research (France); ISCIII - Carlos III Health Research Institute; MRFF - Medical Research Future Fund; NIH - National Institutes of Health (USA); NIHR - National Institute for Health Research; NSF - National Science Foundation (USA); REACTing - REsearch and ACTion targeting emerging infectious diseases; UKRI - UK Research and Innovation 
with UK Research and Innovation (UKRI) ranking first among all funders included in the analysis - more than the combined total of the next four highest-ranked funders. Such an occurrence can, at least partially, be attributed to the stated data limitations of the tracker (see limitations and challenges) whereby UKRI data were relatively more convenient to access and that UKRI is one of the first funders to make data available on repurposed grants.

Of the 15 funders that provided data on known funding amounts, while UKRI invested the most funds according to the latest version of the tracker $(\$ 203.7 \mathrm{~m}$ without taking co-funding into consideration), average grant amounts were largest under the Coalition for Epidemic Preparedness Innovations (CEPI) at $\$ 11.9 \mathrm{~m}$. The Medical Research Future Fund $(\$ 1.4 \mathrm{~m})$ and projects co-funded by the UK's Department for International Development (DFID) and Wellcome $(\$ 1.0 \mathrm{~m})$ were the only other research funders with average awards exceeding $\$ 1 \mathrm{~m}$.

Categorisation of projects against WHO Roadmap priorities \& sub-priorities. All projects were categorised against the priorities and sub-priorities identified by the WHO in their Coordinated Global Research Roadmap, with several research projects being assigned multiple priority and/or sub-priority areas.

WHO Priority Areas. Figure 3 displays both the number of projects listed under each priority area and the known funding amounts (as not all funders provided financial information for their awarded research projects). With the novelty of COVID-19, it is not surprising that the priority area under 'Virus: natural history, transmission and diagnostics' ranks first among all nine priority areas in terms of known funding amounts $(\$ 197.1 \mathrm{~m})$ and second in terms of the number of research projects (408) as researchers seek to improve their basic biological understanding of this new disease and apply that to diagnosis.

While the ranking of most of the priority areas in terms of the number of research projects are relatively consistent with the ranking in terms of known funding amounts, there are three instances where the respective rankings significantly differ: 'Infection Prevention and Control'; 'Candidate Vaccines R\&D'; and 'Social Sciences in the Outbreak Response'. These discrepancies are a reflection of the different grant values for different types of research funded in response to the COVID-19 pandemic. To illustrate this point the average value of 'Candidate Vaccines R\&D' projects (a research area typically associated with high financial costs) was $\$ 1.7 \mathrm{~m}$ - the largest of any priority area. This amount is more than ten-times the average grant amount awarded under the priority area with the smallest average 'Infection Prevention and Control' (\$156k).

The two priority areas with the fewest number of research projects and lowest known funding amounts, 'Animal and environmental research on the virus origin, and management measures at the human-animal interface' (31 projects totalling $\$ 6.4 \mathrm{~m}$ ) and

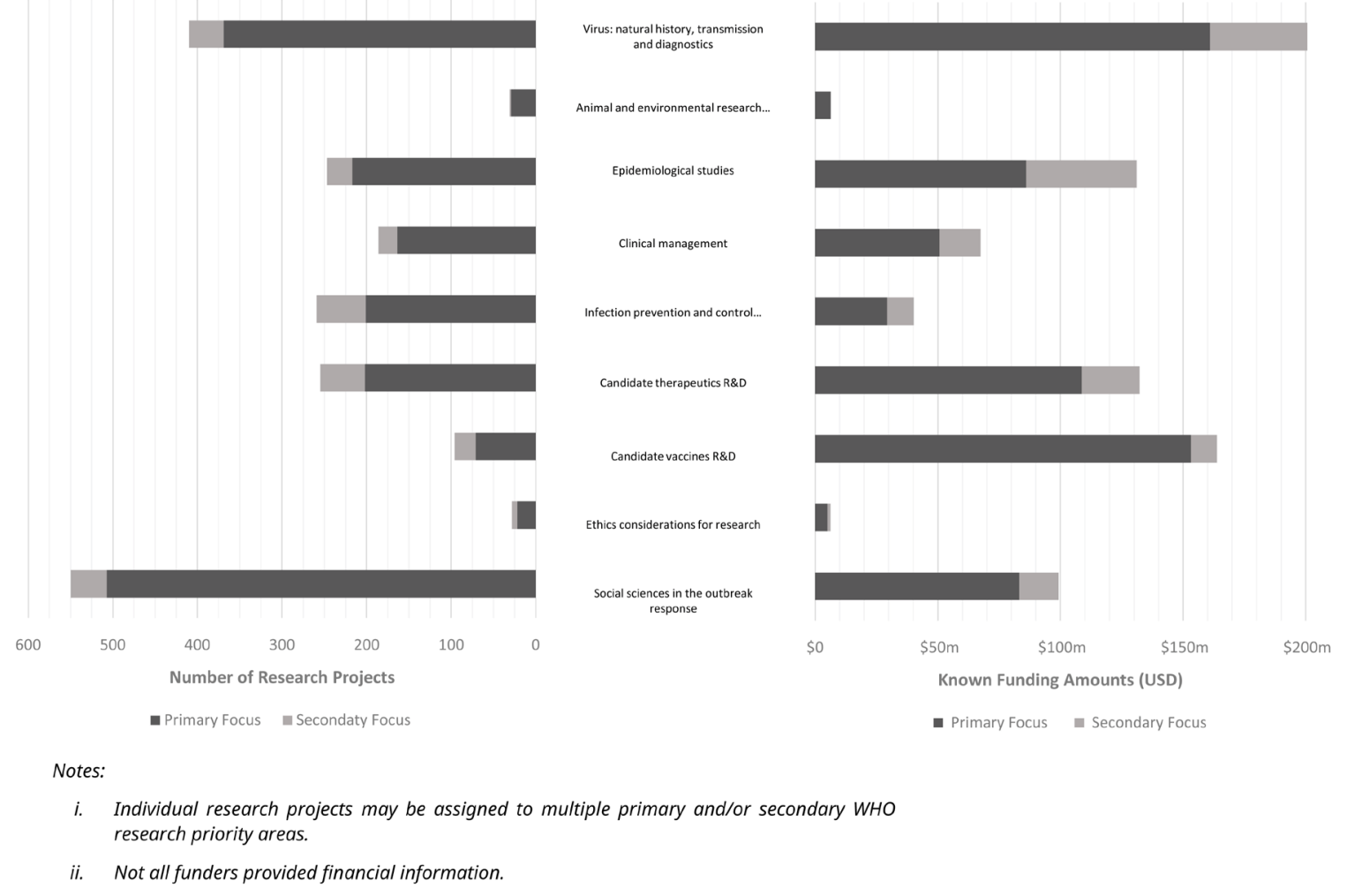

Figure 3. Coronavirus disease 2019 (COVID-19) Research Projects Classified Against Priorities Outlined in World Health Organization (WHO) Coordinated Global Research Roadmap. The latest and previous versions of this figure are available as extended data $^{19}$. 
'Ethics considerations for research' (28 projects totalling $\$ 6.2 \mathrm{~m}$ ) are also the areas with the fewest number of research funders (10 each). This contrasts with the rest of the priority areas that each have between 17 and 22 funders investing in such research.
WHO Sub-Priority Areas. Looking in greater detail, Figure 4 shows how the 1,858 COVID-19 research projects included in the latest version of the tracker have been categorised against abbreviated versions of the 44 sub-priorities mentioned in the

Virus: natural history, transmission and diagnostics

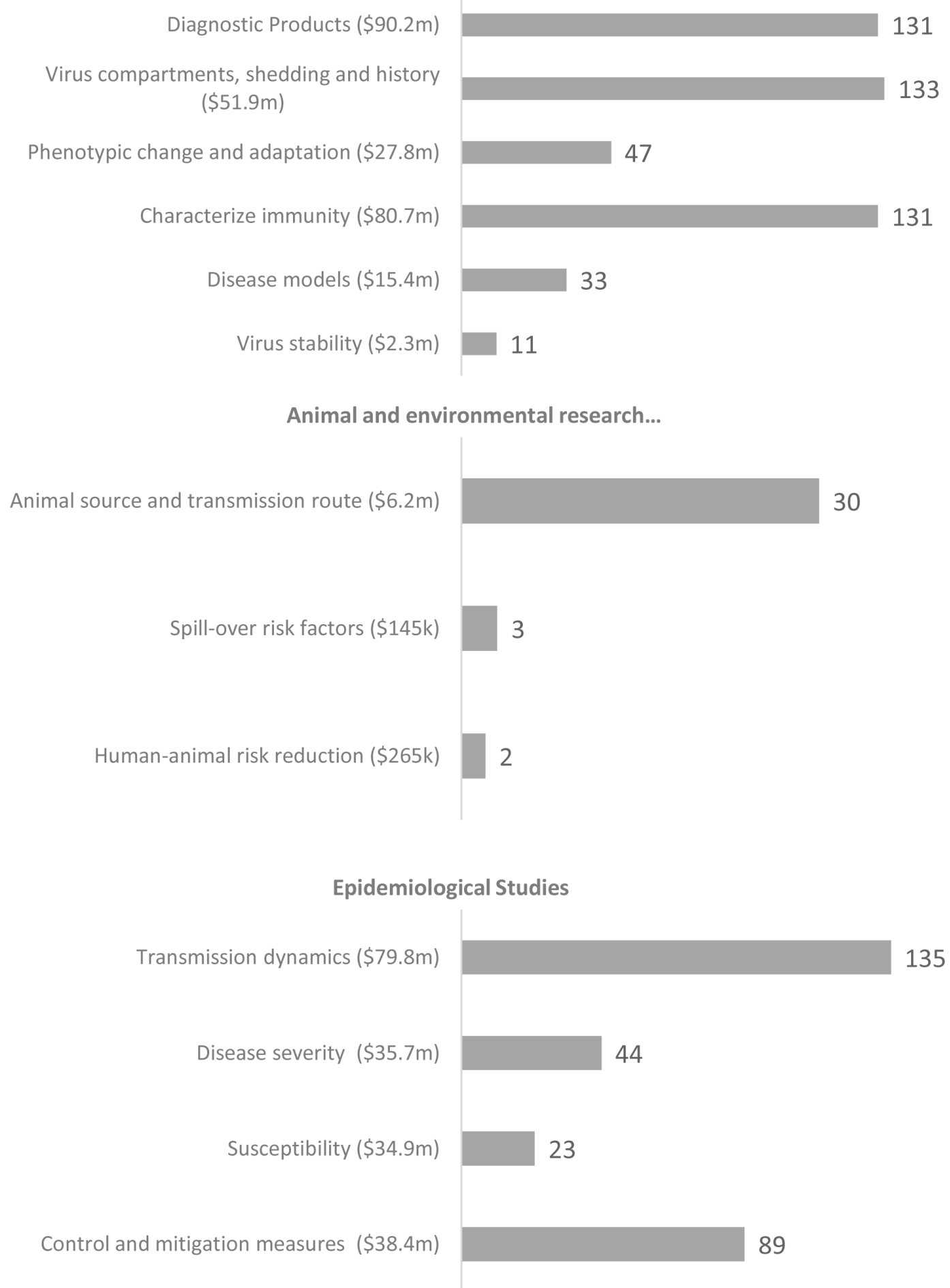




\section{Clinical characterization and management}

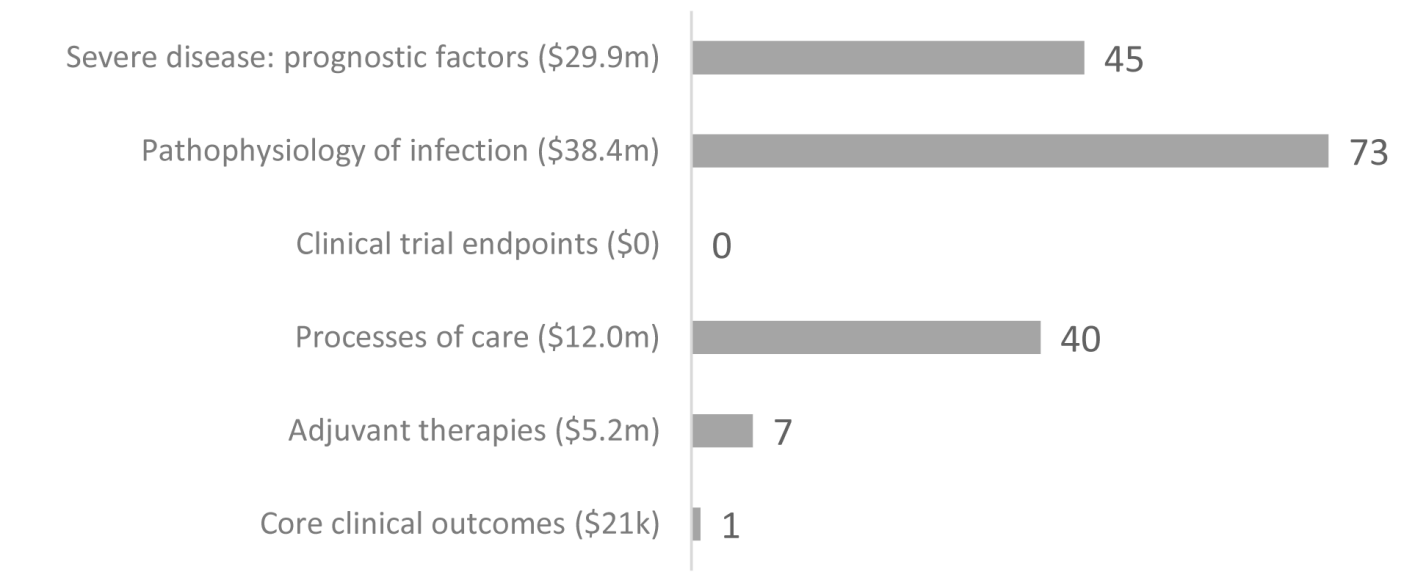

Infection prevention and control, including health care workers' protection

Restriction of movement: effectiveness $(\$ 9.8 \mathrm{~m})$

PPE: effectiveness $(\$ 7.9 m)$

Transmission and the environment (\$12.1m)

Compliance with IPC interventions $(\$ 9.3 \mathrm{~m})$
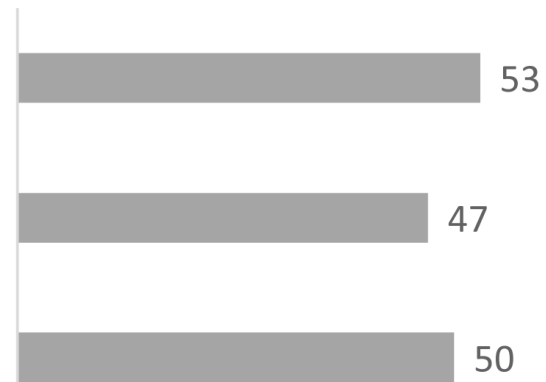

\section{Candidate therapeutics R\&D}

In vitro and vivo testing (\$78.0m)

Prophylactic use $(\$ 11.0 \mathrm{~m})$

Supply of therapeutics (\$356k)

Therapeutics evaluation $(\$ 45.4 \mathrm{~m})$

Combination therapies $(\$ 5.0 \mathrm{~m})$
50

53 


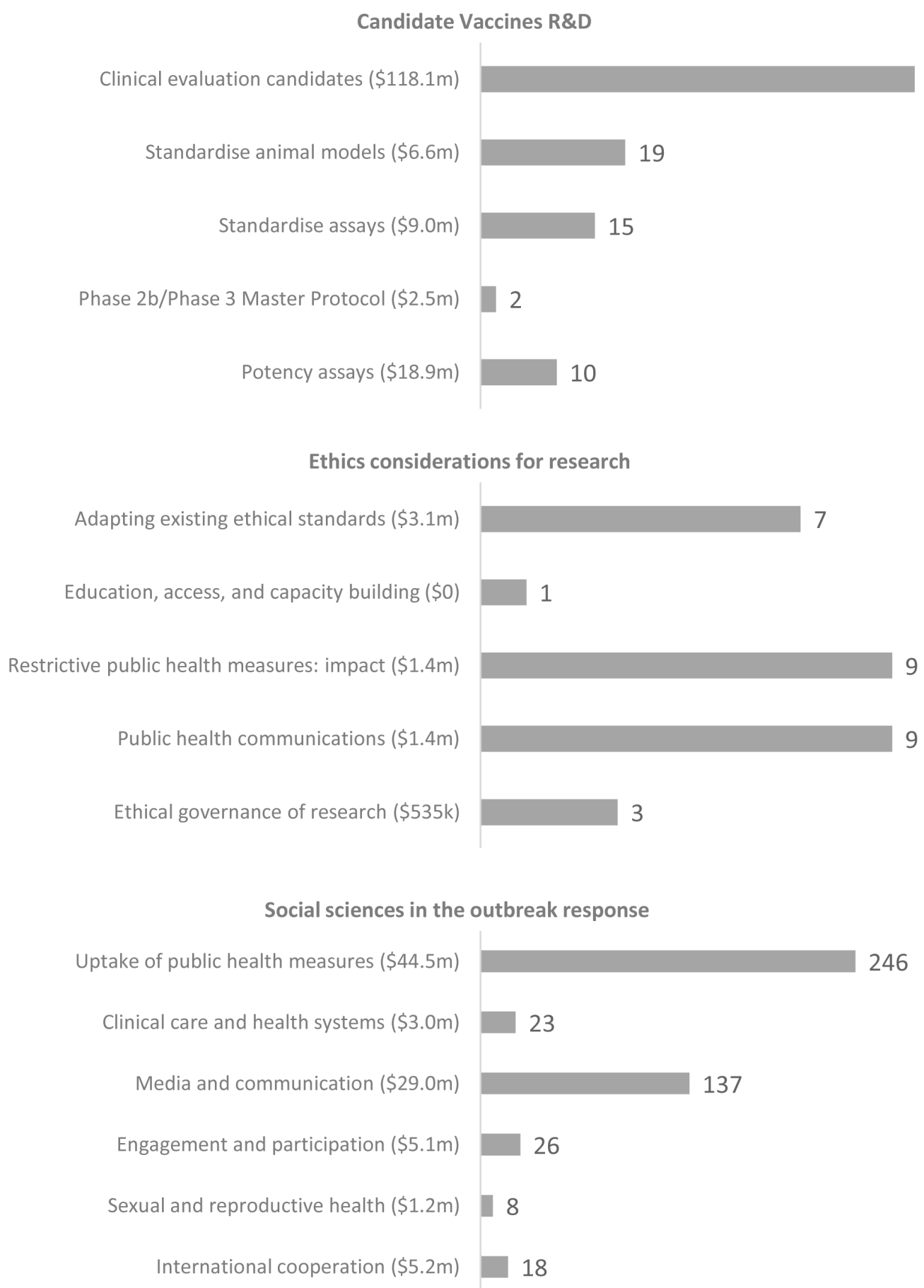

Notes:

i. Individual research projects may be assigned to multiple primary and/or secondary WHO research sub-priority areas.

ii. Funding figures unavailable for $9.6 \%$ of projects included in the latest version of the database as not all funders provided financial information.

Figure 4. Number of research projects included under each sub-priority outlined in World Health Organization (WHO) Coordinated Global Research Roadmap (known funding amounts indicated in brackets). The latest and previous versions of this figure are available as extended data ${ }^{19}$. 
WHO Roadmap. The names of the sub-priorities are listed in full as Extended data ${ }^{19}$.

The funding patterns at the WHO Priority-level are reflected at the sub-priority level. Notably, of the seven sub-priorities with at least 100 awarded research projects, three are from the 'Virus: natural history, transmission and diagnostics' priority and two are from 'Social sciences in the outbreak response'. The sub-priority area with the greatest total investment was "identification of candidates for clinical evaluation in relation to "Candidate vaccines R\&D'". This priority area also features three of the top four sub-priority areas with the largest average funding amounts.

The contrasts, however, in the findings between the priority and sub-priority areas highlight important issues in the distribution of funding for COVID-19 research within priorities. For instance, within the 'Clinical characterization and management' priority area, it can also be seen that the overall priority-level investments concentrated on research addressing only half of the six sub-priority areas. In fact, two of the sub-priority areas ('Optimal endpoints for clinical trials' and 'Develop core clinical outcomes to maximize usability of data across range of trials') rank among the bottom three of all 44 sub-priorities in terms of both number of projects and known funding amounts, but 'optimal endpoints for clinical trials' is the only sub-priority area not addressed by any of the 1,858 COVID-19 research projects in the latest version of the tracker.
More specific research investment gaps emerge within the two priority areas with the lowest levels of investment. Within the priority area 'Animal and environmental research on the virus origin, and management measures at the human-animal interface' just three projects (total value $\$ 145 \mathrm{k}$ ) focused on 'Socioeconomic and behavioural risk factors for spill-over' and only two projects (total value $\$ 265 \mathrm{~K}$ ) address 'Risk reduction strategies at the human-animal environment interface'. For the 'Ethics considerations for research', a single project (no financial information available) has been funded to address the sub-priority on 'Sustained education, access, and capacity building'. While the sub-priority area to 'Promote adequate supply of therapeutics showing efficacy' includes just three projects (total value $\$ 356 \mathrm{k}$ ), this is likely to be a sub-priority that will receive further investment once candidate therapeutics are identified.

Classification of research projects which did not categorise against WHO Roadmap (emergent categories). The majority of research projects which did not categorise against the WHO Roadmap are social sciences research that does not align with the sub-priorities in the WHO Roadmap. These are shown by the newly created categories in Figure 5. These highlight important themes for COVID-19 research, which both researchers and funders are prioritising. 'Mental health' and 'digital health' are the two most prominent emergent categories which do clearly fall within a health remit and it is notable both that these

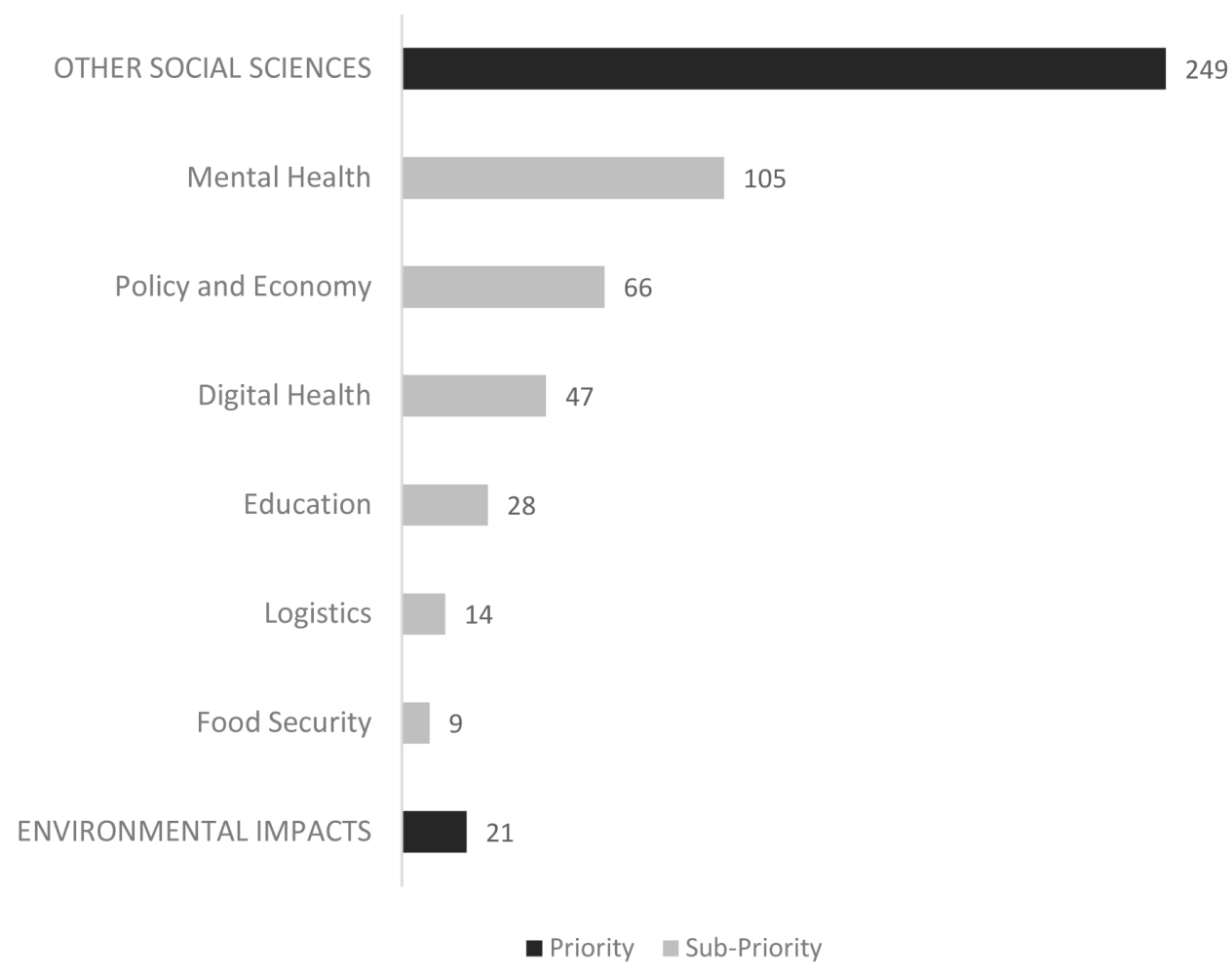

Note: Individual research projects may be assigned to multiple priority and/or sub-priority areas

Figure 5. Number of research projects classified against emergent research priority and sub-priority areas not previously identified. The latest and previous versions of this figure are available as extended data ${ }^{19}$. 
are receiving research attention, but also that currently this is limited to research from a social science perspective, rather than a clinical perspective. The further newly emergent social sciences related sub-priorities of 'policy and economy', 'education', 'logistics' and 'food security' and newly emergent priority of 'environmental impacts' are all focussed on the broader social and economic impacts of the COVID-19 recovery and reflect the broader COVID-19 research focus of the tracker and remits of the funders whose data are currently incorporated.

Location of projects. Figure 6 summarises the location where research projects are taking place. Research is being conducted in 102 countries with the greatest number of projects taking place in the UK ( 825 projects) followed by the United States (609 projects) and then Canada (105 projects).

It should also be noted that, of the 1,858 research projects, 102 $(5.5 \%)$ take place across multiple countries, with research partnerships between Canada and China being the most common (6 projects).

Classifying countries by the Organisation for Economic Co-operation and Development's (OECD) Development Assistance Committee (DAC) list, Figure 6 also shows that nearly all research projects $(93.8 \%)$ are taking place, at least in part, in high-income countries. 148 projects are taking place in at least one of the 67 Official Development Assistance (ODA) recipient countries identified- of which 28 are taking place in the least developed and low-income countries, 20 in lower-middle-income countries and 19 in upper-middle-income countries.

Just 116 projects are taking place in only low- or middleincome countries. Of these, 15 projects are underway in Uganda, followed by Burkina Faso ${ }^{11}$ and South Africa ${ }^{10}$. In total, 11 projects are taking place in China, though we are aware there is much more nationally funded research occurring for which we have not yet been able to obtain data. Among the 116 projects taking place exclusively in low- and middle-income countries, just under a third (30.2\%) are being conducted across multiple countries.

Characteristics of research projects in Low- and Middle-Income Countries (ODA-recipient countries). All research projects in resource-limited settings could be categorised against one or more WHO research priorities. In addition, several were also categorised against the context-specific research priorities outlined identified by the UKCDR, African Academy of Sciences (AAS) and the Global Health Network (TGHN) and are shown in Figure 7 and Figure 8.

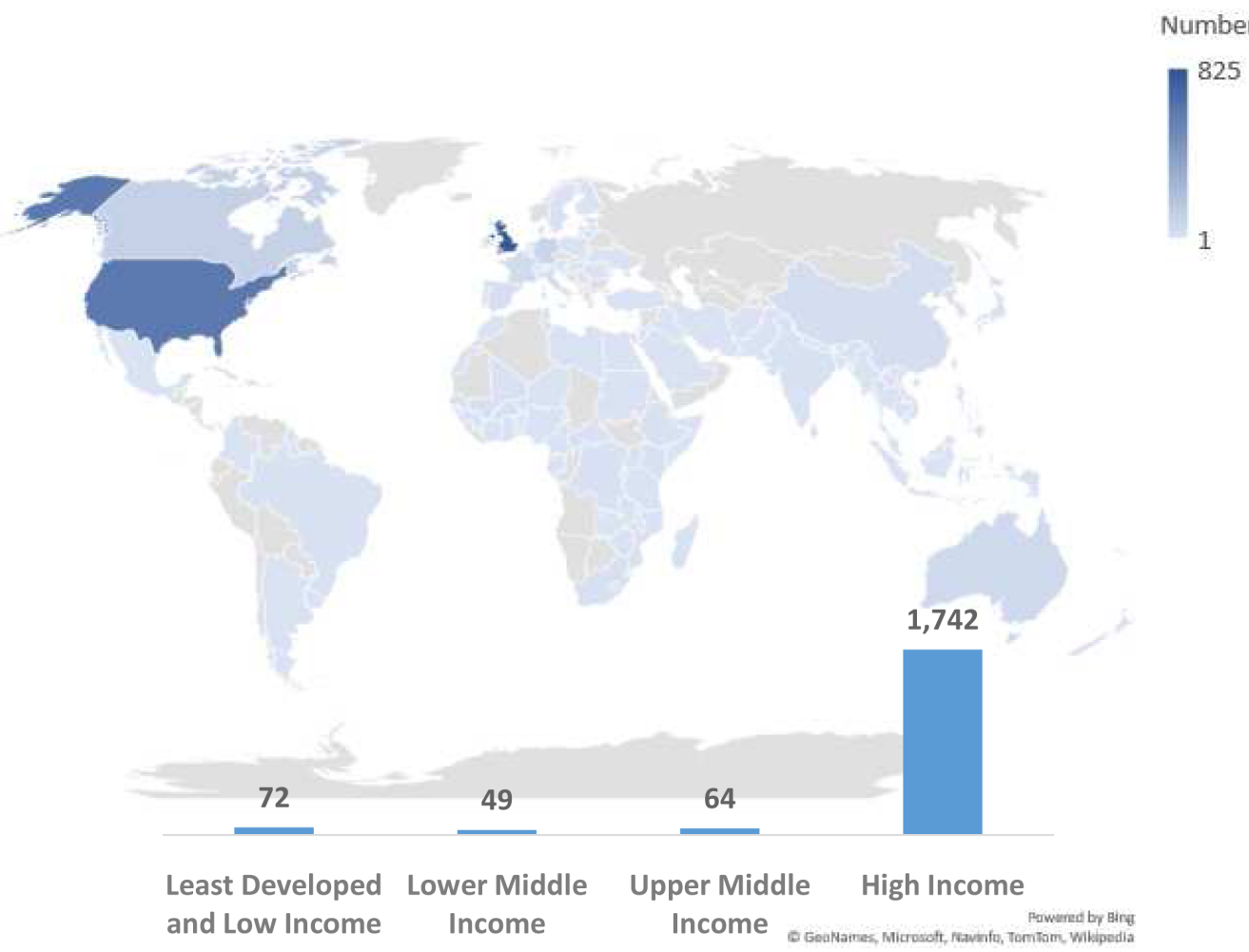

Note: research projects may take place across multiple locations

Figure 6. Location of coronavirus disease 2019 (COVID-19) research projects by country and Organisation for Economic Co-operation and Development's (OECD) Development Assistance Committee (DAC) list categories. The latest and previous versions of this figure are available as extended data ${ }^{19}$. 


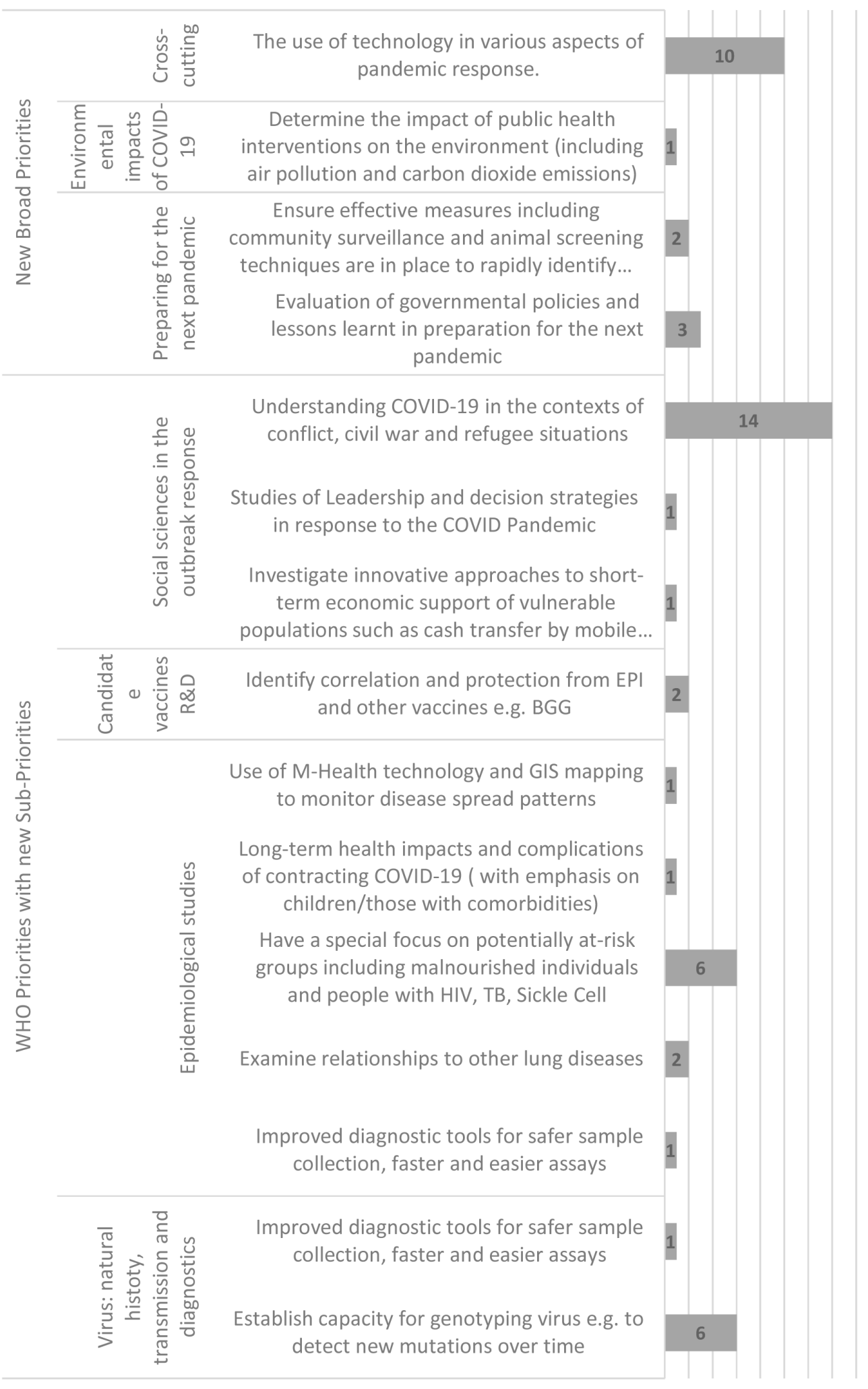

$0 \quad 2 \quad 4 \quad 6 \quad 810121416$

Note: Research projects may be assigned with multiple priority and/or sub-priority areas

Figure 7. Research projects in low- middle-income countries (LMICs) categorised against their research priorities (LMIC Research Priorities). The latest and previous versions of this figure are available as extended data ${ }^{19}$. 

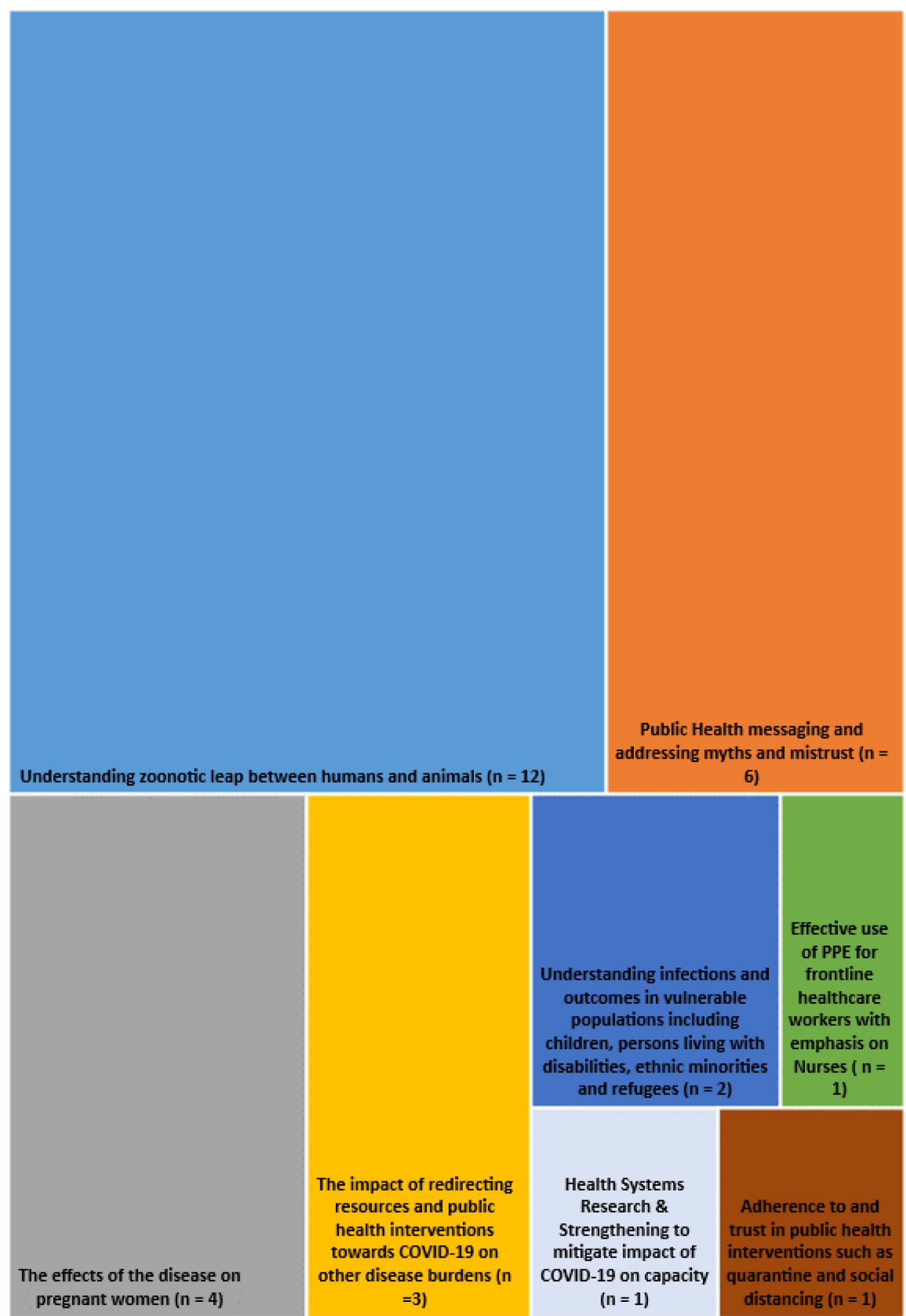

Figure 8. Research projects in low- middle-income countries (LMICs) categorized against 'existing World Health Organization (WHO) Priorities requiring greater research emphasis'. The latest and previous versions of this figure are available as extended data ${ }^{19}$. 
Figure 7 shows that some projects mapped to the context specific sub-priorities under the following WHO priorities: social sciences in the outbreak response; epidemiological studies; virus' natural history, transmission and diagnostics; and candidate vaccines $R \& D$. The predominant theme was research to understand COVID-19 in conflict zones and among refugees and migrant populations whilst research focussed on co-infections and comorbidities such as HIV and tuberculosis and capacity strengthening ranked second. Similarly, a few projects mapped to the new broad priority areas with the highest category being the cross-cutting theme involving the use of technology in various aspects of the pandemic response. Figure 8 shows those projects mapping to existing WHO priorities 'requiring greater research emphasis in LMICs' here the most projects did map to 'understanding zoonotic leap between humans and animals' showing perhaps unsurprisingly that the few projects in the entire tracker focussing on this were taking place in LMICs. In contrast the highlighted priorities of 'Health Systems Research Strengthening to mitigate impact of COVID-19 on capacity' and research on 'Adherence to trust in public health interventions such as quarantine and social distancing' were lacking.
ANRS (France REcherche Nord \& sud Sida-hiv Hépatites French Agency for Research on AIDS and Viral Hepatitis), the European and Developing Countries Clinical Trials Partnership (EDCTP), Canadian Institutes of Health Research (CIHR) and UK Research and Innovation (UKRI) fund the most projects involving LMICs as shown in Figure 9 (bubble diagram). Further, ANRS, CIHR and EDCTP- funded projects are well distributed across the sub-groups of ODA- recipients. Notably, UKRI projects involve comparatively fewer countries since most of their funded projects are concentrated in Uganda and Gambia (the location of two UKRI MRC centres).

The National Science Foundation (NSF) has funded two projects involving China, and the 3 National Institutes of Health (NIH) funded projects span 4 Least Developed Countries. The majority of projects funded by Coalition for Epidemic Preparedness Innovations (CEPI) are in HICs with only 3 in China and India. This likely speaks to the availability of the requisite research capacity in HICs for carrying out preclinical and early stages of vaccine research which these projects are primarily concerned with. It is expected that future updates to the tracker

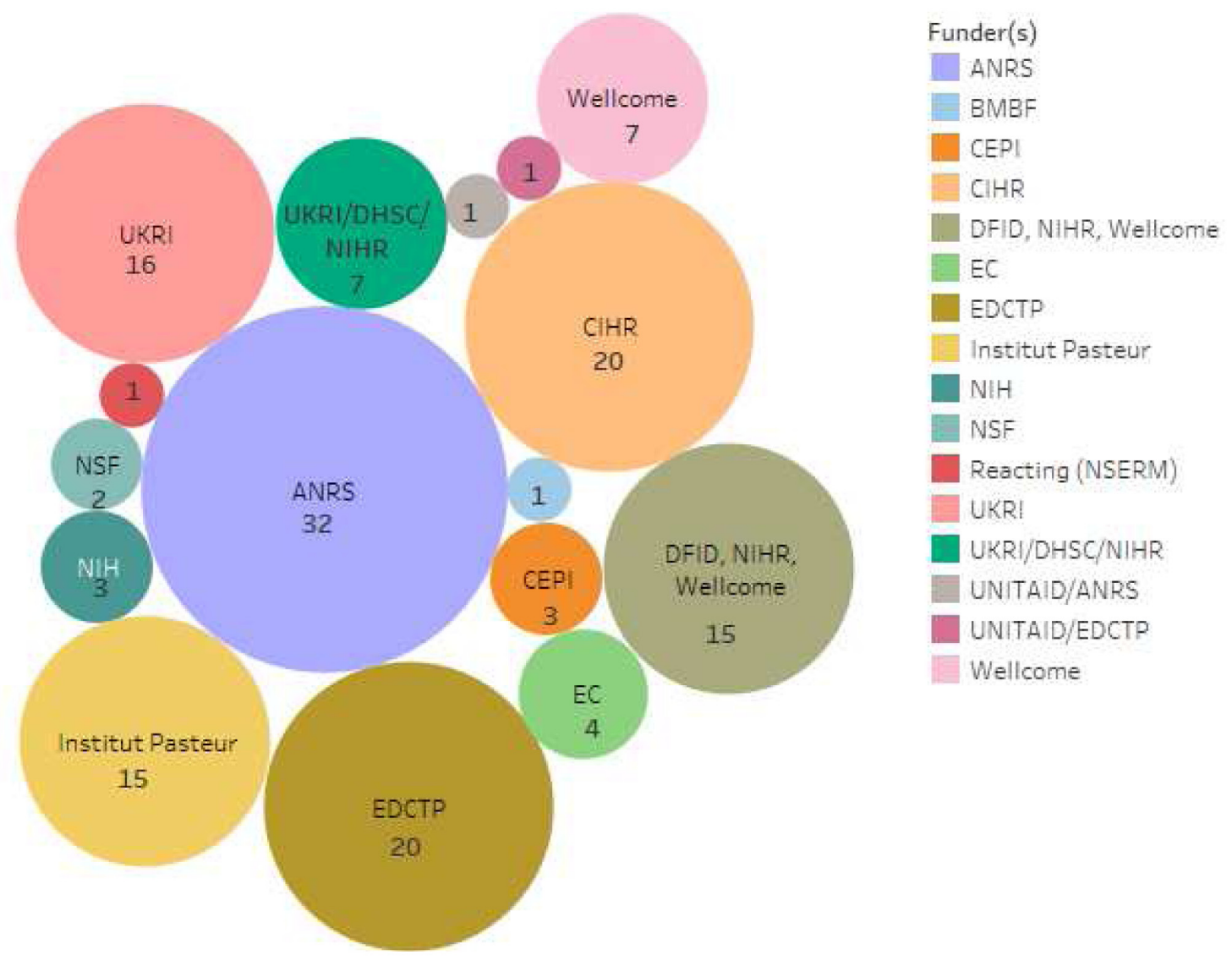

Figure 9. The major funders in Official Development Assistance (ODA)-recipient countries. The latest and previous versions of this figure are available as extended data ${ }^{19}$. 
will feature more research being conducted in LMICs as the outcome of funding calls become known following the increased number of LMIC-specific calls launched by research funders.

Cross-cutting themes. During the review and classification process three additional characteristics of the grants were identified: innovation, repurposed grants and capacity strengthening.

Figure 10 shows the case of the two cross-cutting themes with the greatest number of projects, namely 'innovation' and 'repurposed grants', these figures were mainly driven by the large presence of UKRI data - constituting a significant proportion of the number projects ( $96.7 \%$ and $77.3 \%$, respectively). The NIH was the second major source of repurposed projects, with many projects on other coronaviruses having been repurposed early in the pandemic. The number of repurposed grants in the tracker is expected to increase as funders start to make this data available.

When restricting the analysis to just those coded against cross-cutting themes, the distribution of projects against the WHO priority areas reveal some notable findings in Figure 11. In the case of 'capacity strengthening', much of these projects fell under the priority area of 'Virus: natural history, transmission and diagnostics'(45\%) - nearly double the priority area with the next highest number of projects ('Epidemiological studies' at 25\%). In particular, of these projects, all but two of them have been taking place in, at least, one of 11 African LMICs.

Interestingly, among those repurposed grants, there were two priority areas with significant numbers of projects - namely 'Social sciences in the outbreak response' (38.2\%) and 'Virus: natural history, transmission and diagnostics' $(21.5 \%)$ with all other priority areas being assigned with less than $10 \%$ of repurposed projects. The priority areas most common for those coded against 'innovation' were 'Infection prevention and control' $(21.7 \%)$ and 'Social sciences in the outbreak response' $(20.1 \%)$.

Categorisation of research projects against HRCS categories. To improve our understanding of the type COVID-19 research funded and provide a categorisation that would not change during the course of the pandemic and was comparable to other disease research portfolios, the projects included in the tracker were coded against the research activity codes outlined by the HRCS (Figure 12).

Due to COVID-19 being identified relatively recently, most of the funded research projects included in the latest version of the tracker address the more elementary stages of biomedical and health research, specifically underpinning research $(19.5 \%)$, aetiology $(23.6 \%)$ and the prevention of disease and conditions, and promotion of well-being $(8.6 \%)$.

While only 821 of 1,858 projects have been classified against the HRCS research activity codes, it is anticipated that all research projects will be classified with future updates to this analysis.

Study populations included in projects. Most research projects included in the latest version of the tracker deal with human populations $(56.9 \%)$ with a significant emphasis on populations that have tested positive for COVID-19 (21.5\% of research projects studying human populations). More than one in seven projects $(16.1 \%)$ studying humans is focused on vulnerable

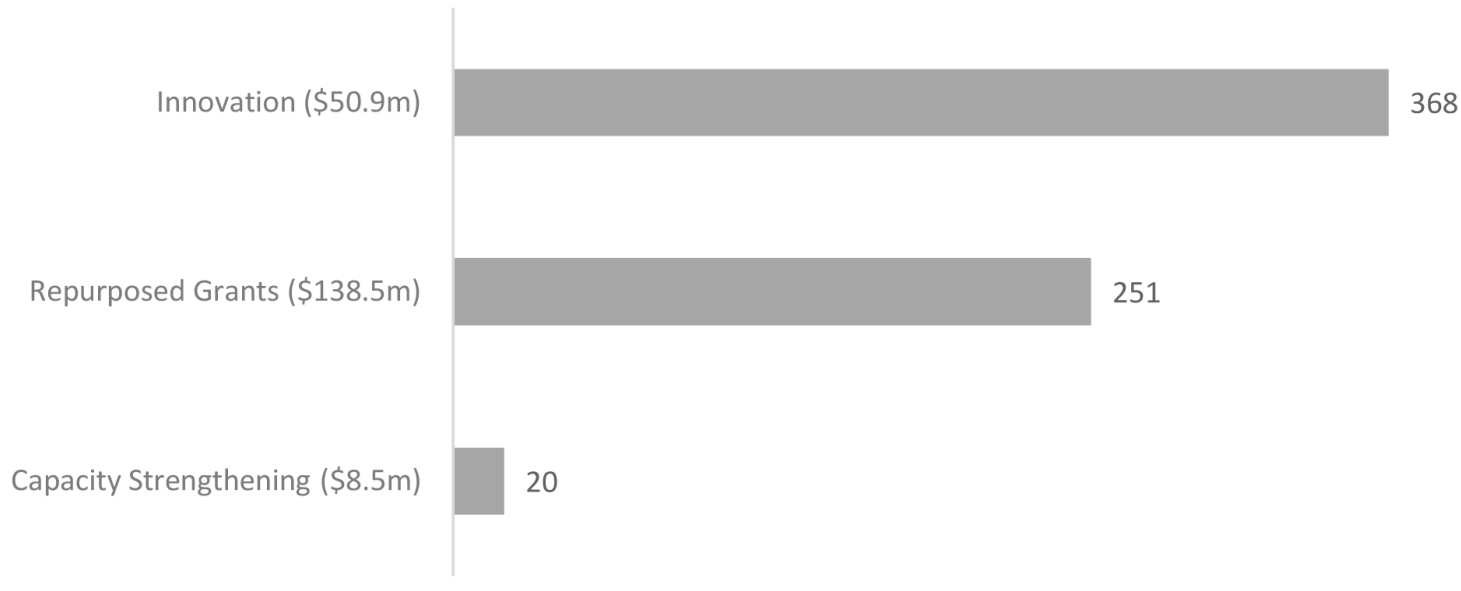

Notes:

i. $\quad$ Research projects may be assigned with multiple cross-cutting themes

ii. Not all funders provided financial information.

Figure 10. Number of research projects included under each cross-cutting theme (known funding amounts indicated in brackets). The latest and previous versions of this figure are available as extended data ${ }^{19}$. 
Virus: natural history, transmission and diagnostics

Animal and environmental research...

Epidemiological studies

Clinical management

Infection prevention and control...

Candidate therapeutics R\&D
Candidate vaccines $R \& D$
thics considerations for research
Social sciences in the outbreak response

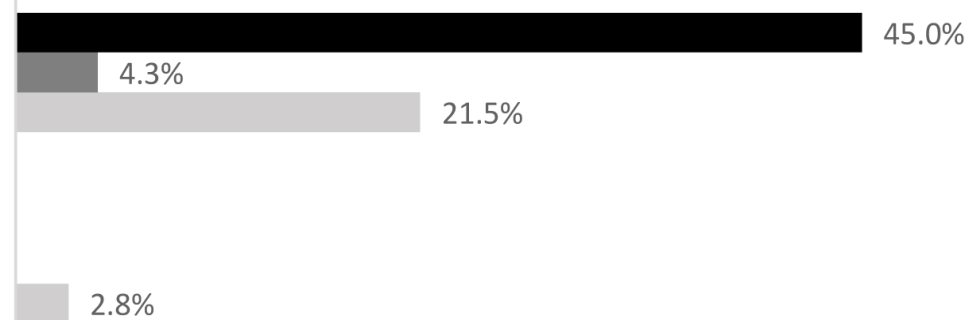

$2.8 \%$

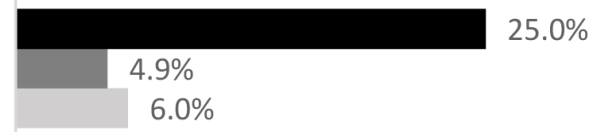

$15.0 \%$

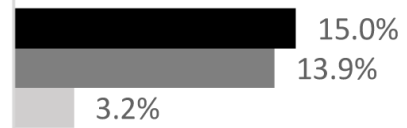

$3.2 \%$
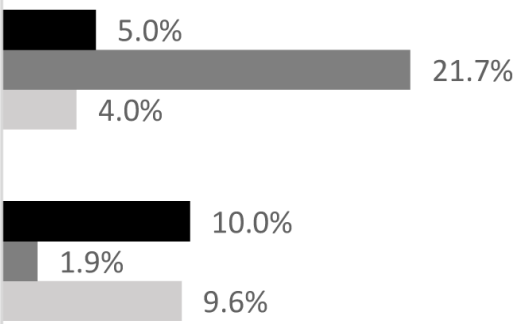

$1.6 \%$

$6.4 \%$

$15.0 \%$

$20.1 \%$

$38.2 \%$

- $\%$ of Capacity Strengthening (20 projects)

- \% of Innovation (368 projects)

— o of Repurposed Grants (251 projects)

Note: Research projects may be assigned with multiple cross-cutting themes

Figure 11. Research projects with cross-cutting themes broken down by World Health Organization (WHO) Priority Area. The latest and previous versions of this figure are available as extended data ${ }^{19}$. 


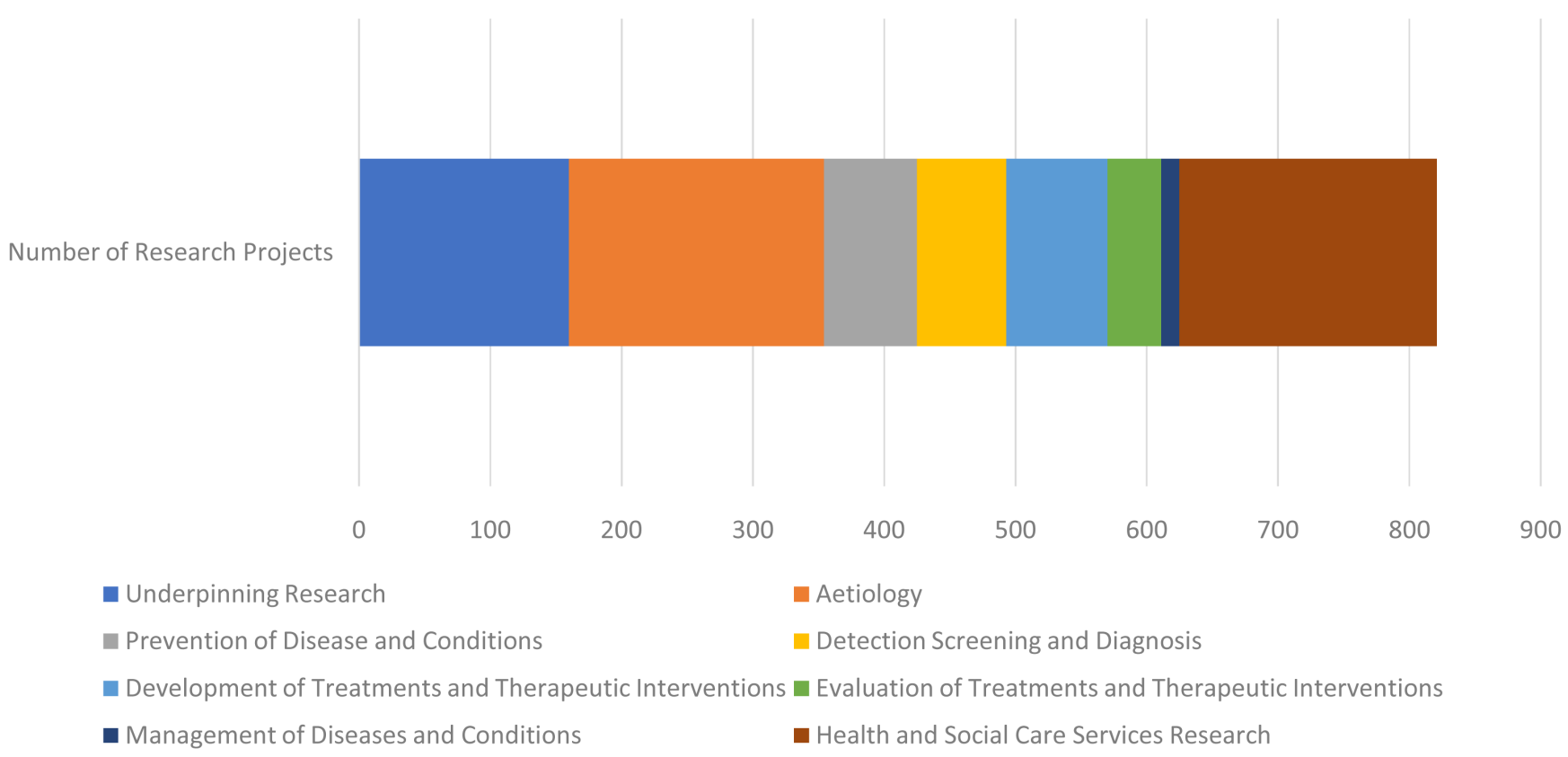

Figure 12. Research activities of coronavirus disease 2019 (COVID-19) research projects classified using health research classification system. The latest and previous versions of this figure are available as extended data ${ }^{19}$.

population groups. Figure 13 summarises how the research projects are classified across all levels of the study population categorisation system outlined above.

\section{Discussion}

This baseline review of the UKCDR and GloPID-R COVID-19 Research Project Tracker has described the huge investment and wide range of research projects repurposed or newly funded related to COVID-19 between January 1 and July 15, 2020. We are keen for researchers, funders and policy makers to engage with these data directly for their areas of specialism and interest, through extracting the relevant data from the tracker and undertaking their own analyses to aid decision making. Given the time demands on all parties in the pandemic, we hope that the regular provision of these descriptive and thematic analyses provide broad insights to help inform the research community and improve the efficiency and effectiveness of the research response going forwards.

\section{Alignment of the funded research portfolio to the WHO Roadmap}

Importantly, here we have aligned the funded research to the mid- long term research and innovation priorities of the WHO Roadmap, and disaggregated the data by locations and population to give a detailed picture of how the research landscape aligns to these global research priorities.

There are gaps in the global research funding portfolio, specifically related to the priority areas of 'Ethics considerations for research' and 'Animals and environmental. We believe that these do represent important and real research gaps, towards which the research community should be shifting its attention.
However, it is also important to note the intention and detail of these and all priority areas within the WHO Roadmap, where there is variability in who is best positioned to address the research sub-priorities with some clearly needing external research activity and others indicating research activity which the WHO planned to undertake directly themselves.

The lack of alignment of funded research projects to the 'Ethics considerations for research' priority may be one such example, as it misses the direct activity that the WHO has undertaken in to address this priority through direct research and provision of important guidance on ethical matters relating to COVID-19 $9^{11}$ which align to the sub-priorities as well as the clear strength of ethical consideration across the majority of research projects (which don't have a core focus on ethics). Despite these considerations, both researchers and research funders need to pay greater attention to the prominence placed on ethical considerations for research by the WHO and ensure that further research is undertaken on those aspects outlined under the roadmap priority area explicitly.

For 'Animals and environmental research', again the WHO is currently undertaking direct activity in this regard (through their developing mission with China to identify the specific source and intermediate pathways of transmission for SARS CoV-2 into humans ${ }^{12}$ ). However, except for a few notable projects in LMICs, the instigation of necessary broader research activity in this area, particularly looking towards gaining broader understanding of how such viruses emerge in the human population and proactive surveillance is certainly limited and needs to be expanded and longer term in nature. This research needs to be undertaken in locations where diseases are 


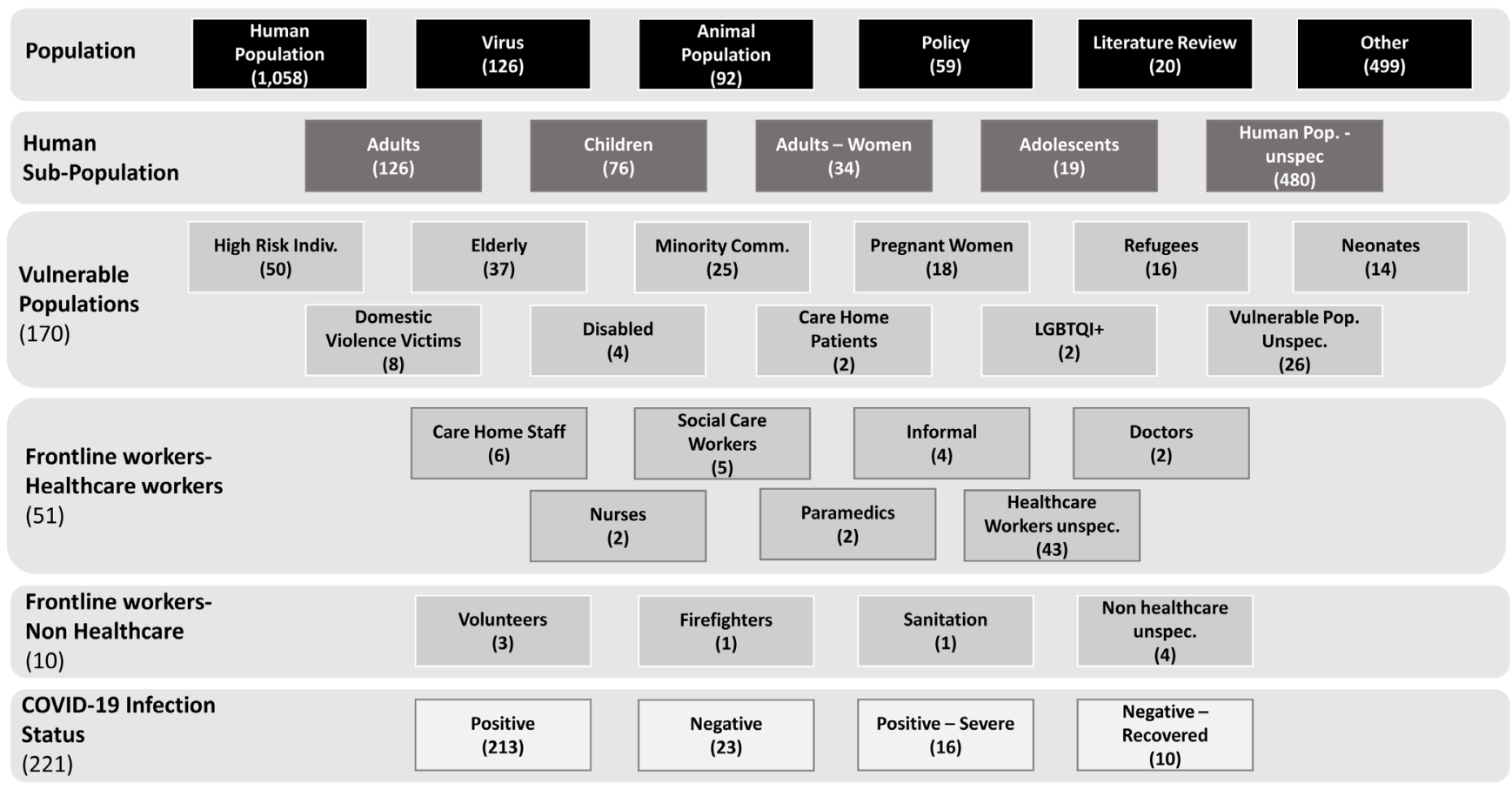

Note: Individual research projects may be classified against multiple categories/sub-categories.

Figure 13. Coronavirus disease 2019 (COVID-19) research projects classified using study population categorisation system (number of projects indicated in brackets). The latest and previous versions of this figure are available as extended data ${ }^{19}$.

most likely to emerge, due to the nature of interactions between humans and animals ${ }^{13}$, many of which are LMICs and this could therefore play an increasingly important role in the research portfolios in these locations going forward. It is also important that this research activity for this priority expands beyond the remit of the WHO and through collaborations with the UN Food and Agriculture Organisation (FAO) and the World Organisation for Animal Health (OIE) through a One Health framework $^{14}$.

Beyond the clear gaps at the priority level, it is inherently difficult to conclude when a particular priority area has received sufficient research funding or research projects at the grant award stage, as this is only apparent when the outcomes are achieved and it is clear that the research question has been sufficiently answered. We do however further note, that within some of the better funded WHO research priority areas, there are still certain sub-priorities which again are clear gaps. This is the case for 'Optimal endpoints for clinical trials' and 'Develop core clinical outcomes to maximize usability of data across range of trials' within the 'Clinical characterisation and management' priority area. This may result from the fact that these activities will be implicit but not explicit in clinical research projects; however, this in itself may indicate a clear issue where both these sub-priorities are essential for collation of results across studies and should therefore be explicit, pointing to the generally observed lack of coordination beyond a few pre-established clinical trial networks as highlighted in discussions at the recent GloPID-R Synergies Meetings ${ }^{15}$. In contrast, the variability of research activity indicated within the 'Candidate therapeutics R\&D' priority area appears to reflect the inter-dependencies of these sub-priorities rather than necessarily a gap needing immediate funding, with research into 'Supply of therapeutics' depending to some extent on the identification of particular safe and effective therapeutics.

For those sub-priorities where research investments have been focused there will be benefits to enhanced coordination. We have already highlighted the wide range of social science research projects addressing 'Uptake of public health measures' and 'Media \& communication' sub-priorities to the WHO COVID-19 Social Sciences working group. The basic virus research on 'Diagnostic products', 'Virus compartments shedding and history' and 'Charactering immunity' are further areas where coordination should be explored globally due to large funded research portfolios.

\section{Location of research}

Most of the funded research projects in the tracker are located in HICs, reflecting national funding by some of the wealthiest research funders during the first phase of this pandemic, with the truly global nature of the pandemic meaning that virus was circulating in these countries to enable relevant clinical research. A large amount of research has also been funded 
within China, although as explained in the limitations we have not managed to incorporate this. The global distribution of funding is now expected to start to shift, with a review of the UKCDR and GloPID-R funders revealing at least eight open or recently closed funding calls specifically related to LMICs. There appears to be a growing recognition that context specific research is needed in $\mathrm{LMICs}^{9}$, although the results presented here show little funding dedicated to context specific research priorities relating to health systems, trust in public health interventions, optimal personal protective equipment use, health care worker support and community engagement. We will continue to expand our analyses in this area as this is an area of focus for COVID CIRCLE.

\section{Research populations}

The disaggregation of research projects by populations is particularly insightful with regards to the 'Social Sciences' WHO Roadmap priority, but also for the 'Clinical management' and 'Epidemiological studies' priority areas. A range of vulnerable populations appear to be well represented for the social sciences including 'minority populations' with recent funding calls in the UK (by UKRI and National Institute for Health Research (NIHR)) having focussed on researching Black, Asian and Minority Ethnic (BAME) populations due to the emerging evidence that they are at higher risk from COVID-19 than white people. A range of health care worker populations and other frontline workers are also included in research funded which again is important due to the clear evidence on greater risks of exposure to individuals in certain occupations ${ }^{16}$ in this pandemic. Children are well represented in the epidemiological studies in accordance with the prioritisation of understanding their role in transmission.

\section{Beyond the WHO Roadmap}

Given the funded research projects within the tracker relate to disciplines beyond health (with relevance to COVID-19) it is unsurprising that several important emergent research themes identified relating to broader social sciences disciplines (policy and economy; education; logistics and food security) and also environmental research, extend beyond the priorities included in the WHO Roadmap Priorities. These all represent important areas for COVID-19 research which funders and researchers are already prioritising with research projects. The two emergent themes of mental health and digital health are however directly relevant to the health research remit and appear to have not been sufficiently covered in the WHO Roadmap document, although projects on these are being funded. We may also be observing the evolution of research priorities from response to recovery and expect to see further examples of this. The expansion of COVID-19 research beyond the original WHO Roadmap document illustrates the wide-reaching social, economic and cultural impacts of the pandemic.

A key strength of this tracker is its breadth and we have therefore undertaken some initial cross-cutting thematic analyses across it here to highlight additional variables that cross- cut disciplines with the inclusion of capacity strengthening, innovation and repurposed grants for this baseline review. The analyses on these themes will be given greater focus in future iterations as greater data becomes available on repurposed grants, which we view as crucial in allowing researchers to respond rapidly within an outbreak.

\section{Conclusion}

In conclusion, we have here provided a detailed baseline review and thematic analysis across the COVID-19 funded research available and we now encourage the research community to use this and the tracker tool to support informed decision making on further research prioritisation going forwards, based on the knowledge of what research is already initiated. We encourage research funders to continue to submit their data to the tracker to ensure it can be as effective as possible.

We have focussed our analysis in this baseline paper on highlighting the clear gaps in the portfolio; however, it is also important to note that for those sub-priorities receiving the most funded projects enhanced coordination would also be beneficial and we will expand on our consideration of these areas in future iterations of this review as the numbers of research projects expand.

We have also shown here the power of tracking research funding at source in real-time, which is particularly important in the fast-moving research environment created by a pandemic, but may have benefits for other global collaborative research efforts going forward. The emerging issue within this pandemic of nationally funded projects resulting in underpowered studies not achieving their aims, means that researchers and funders need to be much more strategic going forwards to efficiently and effectively advance knowledge within epidemics and pandemics.

\section{Limitations of findings and challenges}

To the best of our knowledge we have compiled the most comprehensive database of funded COVID-19 research. We are however very mindful of its inherent limitations and the difficulties in gaining a fully comprehensive picture in what is a truly global research effort to a global pandemic. One main limitation is the absence of commercial research data making inferences on gaps in the vaccine and therapeutics portfolios difficult (this is lacking due to associated intellectual property restrictions). This tracker however has rich data on the early stage development research for those same priorities which is valuable for public funder coordination efforts and enables thematic analyses across disciplines. Another limitation is the fact that few funders to date have shared data on repurposed grants or grants for institutional funding which may have been used for COVID-19 related research.

We are also aware of several funders across wider geographies and disciplines, from whom we have not yet been able to incorporate data. We call here for further research funders (especially within LMICs) to submit their data to make this tracker and associated analyses more accurate to improve the ongoing coordination and help focus limited resources. 
The alignment of research in this tracker to the priorities outlined in the WHO Roadmap also has its challenges, given the Roadmap was produced at speed by drawing together findings from different working groups operating in different ways. The resulting priorities are unsurprisingly imbalanced with some covering much broader research areas than others and with not all sub-priorities intended to be addressed by newly funded research. We have tried to account for this in the discussion of the results here. Another limitation of these priorities and indeed any priorities in a pandemic is their limited temporal nature. The WHO Roadmap priorities that we have mapped here, although named mid- to long-term priorities, were identified by world experts in February 2020, at a time when the majority of cases of COVID-19 were still in China and a pandemic had not yet been declared. We will therefore be updating our mapping as soon as an anticipated new version of the WHO Roadmap is released as research priorities have evolved with increasing knowledge.

\section{Sustainability and future work}

This living systematic review will be updated on a quarterly basis for the duration of the COVID CIRCLE initiative. Future planned work includes incorporation of any new priorities or sub-priorities from the anticipated revision of the WHO Roadmap (following the $1^{\text {st }}-2^{\text {nd }}$ July 2020 meeting). Given the tracker contains a broad range of research relating to COVID-19 (beyond health research) and the evolution towards longer term thinking around research priorities, we are also discussing incorporating coding to the UN COVID Recovery Research Roadmap and are in discussions with the team developing this.

Future iterations will focus in more depth on highlighting areas for potential collaboration in those parts of the portfolio with many funded projects and will identify any trends in funding over time.

\section{Data availability}

\section{Underlying data}

The continuingly updated data related to this study are openly available in the 'COVID-19 Research Project Tracker by UKCDR \& GloPID-R' at https://www.ukcdr.org.uk/fundinglandscape/covid-19-research-project-tracker/.

Harvard Dataverse: Replication Data for Baseline results of a living systematic review for COVID-19 funded research projects. https://doi.org/10.7910/DVN/FBWNTC ${ }^{18}$

This project contains the following underlying data:

- 20200715 Underlying Data Norton et al August 2020.xlsx (Spreadsheet of funded COVID-19 research project)

\section{Extended data}

Figshare: Extended data for Baseline results of a living systematic review for COVID-19 funded research projects. https://doi. org/10.6084/m9.figshare.12847928.v2 ${ }^{19}$

This project contains the following extended data:

- Ext data 1 COVID19 Research Project Tracker_Template_ Norton et al August 2020.xlsx (Template spreadsheet)

- Ext data 2 grant sources Norton et al August 2020.docx (Grant information sources)

- Ext data 3 WHO priorities Norton et al August 2020.docx (WHO COVID-19 research priorities)

- Ext data 4 African and LMIC research priorities Norton et al August 2020.docx (List of African and LMIC research priorities)

- 20200813 - Figure 1 (PRISMA Flow).docx (Baseline review version of Figure 1)

- 20200813- Figures 2 \& 4-12.docx (Baseline review version of Figure $2 \& 4-12$ )

- 20200813- Figures 3 \& 13.pptx (Baseline review version of Figure $3 \& 13$ )

- Baseline Systematic review Tables 1 \& 2.docx (Baseline results Table $1 \& 2$ )

Reporting guidelines

Figshare: PRISMA checklist for 'Baseline results of a living systematic review for COVID-19 funded research projects' https://doi.org/10.6084/m9.figshare.12847928.v2 ${ }^{19}$

Data are available under the terms of the Creative Commons Zero "No rights reserved" data waiver (CC0 1.0 Public domain dedication).

\section{Acknowledgements}

We would like to thank all funders who have provided data to the tracker to date. We thank Laura Scott and Alice Cross from UKCDR for their support to the authors in coding on the tracker. We also thank the Infectious Diseases Data Observatory (IDDO) for inspiring us to undertake this analysis as a living systematic review, through their living systematic review of COVID-19 clinical trial registrations ${ }^{15}$. 
1. World Health Organization: A research and development Blueprint for action to prevent epidemics - Plan Of Action May 2016. 2016; 44. Reference Source

2. World Health Organisation: A Coordinated Global Research Roadmap: 2019 Novel Coronavirus. 2020; [cited 2020 Jul 30].

Reference Source

3. UKCDR \& GloPID-R: COVID-19 Research Project Tracker. 2020; [cited 2020 Jul 30]. Reference Source

4. United Kingdom Collaborative on Development Research (UKCDR): Epidemics Preparedness and Response Group UKCDR. [cited 2020 Aug 1]. Reference Source

5. Norton A, Mphahlele J, Yazdanpanah Y, et al.: Strengthening the global effort on COVID-19 research. Lancet. 2020; 396(10248): 375. PubMed Abstract | Publisher Full Text | Free Full Text

6. https://www.ukcdr.org.uk/about-us/our-members/

7. https://wwww.glopid-r.org/about-us/members/

8. African Academy of Sciences: Research and Development goals for COVID-19 in Africa The African Academy of Sciences Priority Setting Exercise. Reference Source

9. Norton A, De La Horra Gozalo A, Feune de Colombi N, et al.: The remaining unknowns: a mixed methods study of the current and global health research priorities for COVID-19. BMJ Glob Health. 2020; 5(7): e003306. PubMed Abstract | Publisher Full Text | Free Full Text

10. HRCS: UKCRC Health Research Classification System. 2020; [cited 2020 Jul 30].

Reference Source

11. Ethics and COVID-19. World Health Organisation. [cited 2020 Aug 10]. Reference Source
12. World Health Organisation Media Briefing on COVID-19. $7^{\text {th }}$ July 2020. [cited 2020 August 15].

Reference Source

13. Dobson A, Pimm S, Hannah L, et al.: Ecology and economics for pandemic prevention. Science. 2020; 369(6502): 379-381. PubMed Abstract

14. Operational Framework for Strengthening Human, Animal and Environmental Public Health Systems at their Interface. World Bank \& Eco Health Alliance Report. Reference Source

15. GloPID-R COVID-19 Research Synergies Meetings: Meeting summaries and recordings.

Reference Source

16. UK Office for National Statistics: Which occupations have the highest potential exposure to the coronavirus (COVID-19)? [cited 2020 August 15]. Reference Source

17. Maguire BJ, McLean ARD, Rashan S, et al:: Baseline results of a living systematic review for COVID-19 clinical trial registrations [version 1; peer review: 1 approved]. Wellcome Open Res. 2020; 5: 116. Publisher Full Text

18. Norton A, Bucher A, Antonio E, et al.: Replication Data for Baseline results of a living systematic review for COVID-19 funded research projects. Harvard Dataverse, V1. 2020 http://www.doi.org/10.7910/DVN/FBWNTC

19. Norton A, Bucher A, Antonio E, et al:: Extended data for Baseline results of a living systematic review for COVID-19 funded research projects. figshare. Figure. 2020.

http://www.doi.org/10.6084/m9.figshare.12847928.v2 


\section{Open Peer Review}

\section{Current Peer Review Status:}

\section{Version 1}

Reviewer Report 21 October 2020

https://doi.org/10.21956/wellcomeopenres.17862.r40581

(C) 2020 Smith P. This is an open access peer review report distributed under the terms of the Creative Commons Attribution License, which permits unrestricted use, distribution, and reproduction in any medium, provided the original work is properly cited.

\section{Peter Smith}

MRC Tropical Epidemiology Group, London School of Hygiene and Tropical Medicine, London, UK

This assembly of the database on which this "systematic review" is based clearly represents a major effort. The database is likely to be most useful to funders, who will wish to avoid supporting research that is unnecessarily duplicative and it will also be of value to researchers seeking to identify others who are working in a similar area or to identify research areas that seem to have been relatively neglected. The authors have gathered data on research supported by 25 funders, mostly based in high income countries. How complete this is, even for these funders is difficult to judge, but based on the projects supported by each funder, there seems to be a strong bias towards UK funders. It is surprising, for example, to see the US NIH having supported less than $25 \%$ of the number supported by UKRI. A notable deficiency in the database, acknowledged by the authors, is the lack of data from China, where there has been substantial research on SARS-CoV-2, and LMICs in general (e.g. Brazil, India). However, it is understandable that there are challenges in accessing comprehensive information from these sources. Another challenge is that most, but not all, of the information presented relates to the number of projects supported rather than the magnitude of the support. The data base is also missing information from private companies, including pharma companies, where much research on vaccines, therapeutics and diagnostics is likely to be supported. It is also unclear whether the databases of registered clinical trials have been trawled to identify ongoing research. For these reasons, I am not convinced that it is appropriate to label the paper as a systematic review as it seems to be a review only of what has been accumulated in the database to date.

I recognize that the database is early in its life, but as an ongoing check on completeness it may be useful, in the future, to link published papers on SARS-CoV-2 to the database to see what is not being picked up in the database, acknowledging that it is not infrequently difficult to link a particular paper to a particular research grant.

In the review, projects in the database are linked to WHO Blueprint list of research priorities defined in February 2020. This helps identify areas where there are possible funding gaps. However, there are some limitation in using the data base for this purpose. For example, the authors note that there are clear gaps in support for 'optimal endpoints for clinical trials and core 
clinical outcomes' and on 'ethical considerations for research'. Both of these areas have had considerable attention, the former in the context of treatment and vaccine trials (the authors acknowledge the problems in identifying research that may be embedded in other research undertakings) and much of the ethical work that has been done has not required specific funding.

Despite some of the deficiencies identified above, the generation of the database has been a valuable undertaking, and as more funders come on board it will become even more useful. Similarly, the ongoing systematic review will give a useful summary of what is in the database and will help identifying trends in funding and areas where support has been lacking.

\section{Are the rationale for, and objectives of, the Systematic Review clearly stated?}

Yes

Are sufficient details of the methods and analysis provided to allow replication by others? Yes

Is the statistical analysis and its interpretation appropriate?

Not applicable

Are the conclusions drawn adequately supported by the results presented in the review? Yes

Competing Interests: No competing interests were disclosed.

I confirm that I have read this submission and believe that I have an appropriate level of expertise to confirm that it is of an acceptable scientific standard.

Author Response 30 Nov 2020

Alice Norton, UK Collaborative on Development Research, London, UK

Thank you very much for your helpful comments on our Baseline paper, we are pleased that you believe that this has been a valuable undertaking. We have now updated the review with the latest data in the tracker up to the $15^{\text {th }}$ October and have also taken that opportunity to address some of your comments within the new version of the paper. This continues (in our view) to be the most comprehensive data set on research funded on COVID-19 with now over five thousand projects from 72 funders (all categorised to the WHO R\&D Blueprint). We have now provided further details relating to magnitude of funding commitment in addition to numbers of projects in the figures relating to funders and agree that this helps with interpretation. We have noted and agree with the limitations that you highlight regarding the challenges in accessing the full breadth of global research data in this field and we have already clearly acknowledged these limitations. We also agree that the 'systematic' terminology may not therefore be fully appropriate for this endeavour and have now instead re-categorised this as a living mapping review. We also agree with your points on the particular sub-priorities in the roadmap which may have received attention outside of newly funded or even repurposed grants, and we have further clarified our discussion of these. We are continuing to expand the global representation of funders in 
the database and are exploring connections with both clinical trial registrations and outputs (although neither of these are straightforward). We hope that you find this latest version of the paper of renewed interest and relevance.

Competing Interests: No competing interests were disclosed.

Reviewer Report 21 September 2020

https://doi.org/10.21956/wellcomeopenres.17862.r40348

(C) 2020 Vaughn D. This is an open access peer review report distributed under the terms of the Creative Commons Attribution License, which permits unrestricted use, distribution, and reproduction in any medium, provided the original work is properly cited.

\section{David Vaughn}

Bill and Melinda Gates Foundation, Seattle, WA, USA

This is a well-written report by the UKCDR and GloPID-R on their living systematic review (Project Tracker) of COVID-19 R\&D including 1858 projects supported by 25 funders across 102 countries as of 15 July 2020 aligning said R\&D efforts with the WHO global research roadmap for COVID-19 R\&D published in March 2020. The tracker also identifies research objectives not included in the WHO roadmap (seven new areas) that may influence planning for future pandemics (including future WHO roadmaps). A spin-off of the effort (COVID CIRCLE) focuses on R\&D in resource-limited settings.

The Tracker allows the global health community to assess the COVID-19 research portfolio to identify gaps (Animal and environmental research? Clinical trial endpoints and outcomes?) and to reduce duplication (more than 300 vaccines being advanced?); reduction of excessive duplication is important given limited global R\&D resources.

The authors are transparent concerning the limitations of the effort. The data is skewed to GloPID$\mathrm{R}$ members. It is heavily UKRI biased. The US NIH, in particular, seems under-represented. Repurposed funded research may be missed. There is limited data from a number of governments (e.g., China as noted by authors) and private sources (e.g., companies, private foundations such as the BMGF, and ethics think tanks). This publication may encourage more LMICs to participate in the accounting.

Is it possible to comment on measured or perceived impacts? Who uses? Is there documentation of times accessed? Is it timely information if the figures are updated only every three months? The group should conduct a survey at the end of the calendar year to see if funders changed direction based upon this effort. Has there been a reduction of duplication? Or, will this be a retrospective of what happened with little real-time impact on what was happening? Shifting of funding to LMICs is one positive example of impact. Again, we will need a later assessment to build upon this baseline review focusing on impact on the pandemic response. Has the scope been too broad? 
Are the rationale for, and objectives of, the Systematic Review clearly stated? Yes

Are sufficient details of the methods and analysis provided to allow replication by others? Yes

Is the statistical analysis and its interpretation appropriate?

Not applicable

Are the conclusions drawn adequately supported by the results presented in the review? Yes

Competing Interests: No competing interests were disclosed.

Reviewer Expertise: Vaccine development.

I confirm that I have read this submission and believe that I have an appropriate level of expertise to confirm that it is of an acceptable scientific standard.

Author Response 30 Nov 2020

Alice Norton, UK Collaborative on Development Research, London, UK

Thank you very much for your very helpful comments on our baseline paper. We are delighted to now provide a three-month update paper in which the number of projects and funders has greatly expanded over the interval (to over 5 thousand projects from 72 funders). We aim to continue to achieve further geographical cover through ongoing efforts to raise the profile of the tracker and source data from further funders. Thank you for your suggestion regarding inclusion of data on use of these data and perceived impact, we have now included reference to this in this three-month update. We will also be undertaking a retrospective consultation on the influence of this work as part of the learning element of COVID CIRCLE in early 2021. We hope that you find this latest version of the paper of renewed interest and relevance and we would also like to highlight the more frequent updates and highlights available on the funding tracker website.

Competing Interests: No competing interests were disclosed. 